ARTICLE

\title{
Gene editing with CRISPR-Cas12a guides possessing ribose-modified pseudoknot handles
}

\author{
Eman A. Ageely', Ramadevi Chilamkurthy², Sunit Jana3,6, Leonora Abdullahu³,6, Daniel O'Reilly ${ }^{3,5}$, \\ Philip J. Jensik ${ }^{4}$, Masad J. Damha (1) ${ }^{3 凶}$ \& Keith T. Gagnon (1) ${ }^{1,2 凶}$
}

CRISPR-Cas12a is a leading technology for development of model organisms, therapeutics, and diagnostics. These applications could benefit from chemical modifications that stabilize or tune enzyme properties. Here we chemically modify ribonucleotides of the AsCas12a CRISPR RNA 5' handle, a pseudoknot structure that mediates binding to Cas12a. Gene editing in human cells required retention of several native RNA residues corresponding to predicted 2 '-hydroxyl contacts. Replacing these RNA residues with a variety of ribose-modified nucleotides revealed 2'-hydroxyl sensitivity. Modified 5' pseudoknots with as little as six out of nineteen RNA residues, with phosphorothioate linkages at remaining RNA positions, yielded heavily modified pseudoknots with robust cell-based editing. High trans activity was usually preserved with cis activity. We show that the $5^{\prime}$ pseudoknot can tolerate near complete modification when design is guided by structural and chemical compatibility. Rules for modification of the $5^{\prime}$ pseudoknot should accelerate therapeutic development and be valuable for CRISPR-Cas12a diagnostics.

\footnotetext{
${ }^{1}$ Department of Chemistry and Biochemistry, Southern Illinois University, Carbondale, IL, USA. ${ }^{2}$ Department of Biochemistry and Molecular Biology, School of Medicine, Southern Illinois University, Carbondale, IL, USA. ${ }^{3}$ Department of Chemistry, McGill University, Montreal, Canada. ${ }^{4}$ Department of Physiology, School of Medicine, Southern Illinois University, Carbondale, IL, USA. ${ }^{5}$ Present address: RNA Therapeutics Institute, University of Massachusetts Medical School, Worcester, MA, USA. ${ }^{6}$ These authors contributed equally: Sunit Jana, Leonora Abdullahu. ${ }^{\bowtie}$ email: masad.damha@mcgill.ca; ktgagnon@siu.edu
} 
C lustered regularly interspaced short palindromic repeats (CRISPR) and their CRISPR-associated (Cas) enzymes represent an ancient bacterial immune system evolved to fight invading viral pathogens ${ }^{1-8}$. The core enzymatic component is a ribonucleoprotein (RNP) comprised of one or more Cas proteins, with at least one being a nuclease, which are associated with one or two CRISPR RNAs (crRNAs) ${ }^{7-18}$. After recognition of a short protospacer adjacent motif (PAM) sequence by the Cas endonuclease, base-pairing of crRNA nucleotides to target DNA then guides sequence-specific and site-specific endonucleolytic cleavage of DNA phosphodiester bonds ${ }^{10,19-21}$.

Cas9 from Streptococcus pyogenes (SpCas9) and Cas12a, also known as Cpfl, from Acidominococcus species (AsCas12a) are among the most heavily co-opted and engineered CRISPR-Cas systems for creation of model organisms and development of human therapeutics ${ }^{22-33}$. They both efficiently induce DNA double-strand breaks and guide genome editing in mammalian cells $^{16,22,23,33-38}$, utilize a single Cas enzyme and can function with a single-guide RNA (sgRNA), although Cas9 functions as a dual-guide RNA in nature $8,16,23,33$. While these enzymes can ultimately result in similar gene editing outcomes, such as genetic knockout or donor DNA sequence knock-in, they are quite distinct. Cas12a is a smaller protein that naturally utilizes a single crRNA guide (39 nucleotides), less than half the size of the commonly used artificial Cas9 sgRNA (99 nucleotides) ${ }^{16,20,39}$. Cas12a recognizes thymine (T)-rich PAMs, whereas SpCas9 uses guanine $(\mathrm{G})$-rich $\mathrm{PAMs}^{8,16,21,40}$. The Cas12a crRNA adopts a pseudoknot structure at its $5^{\prime}$ end that creates a "handle" for Cas12a to bind, as opposed to a series of stem structures for SpCas9 $9^{20,39}$.

A unique property of Cas12a is trans activity, which is a nonsequence-specific single-stranded DNA cleavage (ssDNase) activity with high catalytic turnover ${ }^{41,42}$. Trans activity requires that the Cas12a RNP first bind to and cut a target DNA strand, which is referred to as cis activity. Trans activity has been exploited to create molecular diagnostics that can detect very small amounts of DNA and amplify the detection signal through cleavage of ssDNA substrates that can be detected, such as by fluorescence, antibodies on test strips, and nanopores ${ }^{41,43,44}$. The digestion of the ssDNA reporter by trans activity is intrinsically tied to the cis cleavage of the specific target DNA because both mechanisms use the same catalytic residues ${ }^{45,46}$. Mutation of neighboring amino acids or REC (recognition lobe) linker and lid regions of Cas12a, however, could allow trans but not cis activity when Cas12a was bound to a complementary single-strand DNA target ${ }^{46}$. However, it appears that cis and trans activity cannot be fully uncoupled for recognition of double-stranded targets via trans activity without cis cleavage of the target strand ${ }^{45,46}$.

CRISPR-Cas systems hold tremendous promise for revolutionizing medicine, particularly through improved gene therapy or gene-targeted approaches ${ }^{26,47-56}$. To achieve this potential, however, various challenges must be overcome. These include long-term safety, delivery, and efficacy $26,37,52-54,56-59$. Lipid nanoparticles are a leading technology being developed for delivery of Cas enzymes, either as protein or as mRNA that encode the Cas protein, along with their cognate crRNA guides $54,60-64$. In principle, this may allow greater spatial and temporal control over tissue and cell targeting ${ }^{63}$ and provide a better window of safety when compared to therapeutic delivery approaches that involve genetically encoded expression of the CRISPR-Cas system, such as adeno-associated viruses ${ }^{64}$. However, RNA is not an ideal drug due to its size and lability-it is quickly degraded in the body. Chemical modification has emerged as a practical necessity to stabilize and control the halflife, bioavailability, and activity of RNA and nucleic acid drugs $^{65,66}$. Chemical modification has been a pillar in the growing success of other nucleic acid-based therapeutics, including antisense oligonucleotides (ASOs) and small interfering RNAs (siRNAs) ${ }^{58,66,67}$. The lessons learned from these technologies can be applied to CRISPR-based therapeutics. The degree of chemical modification required for crRNA guides is unclear and may depend on delivery or target tissue. Nonetheless, ultimate control over stability, protein interaction, enzyme activity, and pharmacology would ideally be achieved by understanding what chemical modifications are tolerated at every position within a crRNA guide $^{47,61,66,68-71}$.

Previous investigations of CRISPR-Cas guide RNA chemical modification have primarily focused on improving therapeutic gene editing by increasing nuclease resistance, understanding biochemical limitations, and reducing off-target editing. Minimal addition of modified nucleotides or inter-nucleotide linkages at the guide RNA $5^{\prime}$ and $3^{\prime}$ termini have been shown to maintain or increase editing, presumably through reduced nuclease sensitivity. These have included $2^{\prime}$-O-methyl with phosphorothioate (PS-2' $\mathrm{OMe})$ or $2^{\prime}$-O-methyl-3'-thiophosphonoacetate (2'OMe thioPACE) for Cas $9^{72,73}$. In the Cas12a system, $3^{\prime}$ end capping has been explored with $2^{\prime}$-F, 2'-O-methyl, unlocked nucleic acid (UNA), locked nucleic acid (LNA), and phosphorothioate (PS) ${ }^{74}$ while $5^{\prime}$ end capping has been explored by addition of short MS or DNA-PS modified sequences onto the $5^{\prime}$ handle ${ }^{75}$. Interestingly, extension of the $3^{\prime}$ end with $2^{\prime}$-O-methyl nucleotides ${ }^{76}$ or the $5^{\prime}$ handle with DNA-PS ${ }^{77}$ has also been reported to improve editing or increase the kinetics of trans cleavage activity for Cas12a, respectively.

More complete or systematic modification of Cas9 and Cas12a guide RNAs has been explored using a variety of chemistries. DNA ( $2^{\prime}$-deoxy) has been reported to alter enzyme activity or specificity while helping to probe $2^{\prime}$-hydroxyl requirements of the enzyme ${ }^{69,78-80}$. A broader array of modifications, like $2^{\prime}-\mathrm{F}, 2^{\prime}-O$ methyl, bridged nucleic acid (BNA), UNA, LNA, $2^{\prime}$ F-arabinonucleic acid (FANA), and PS have been tested alone or in combination as well $61,70,71,81-84$. These studies largely demonstrated that much of the guide could be modified, with moderate to high editing preserved, but particular positions, such as seed regions or individual residues, required RNA nucleotides. Specificity could also be improved with careful placement of modifications in the guide region $78,79,83,84$. For Cas 9 , retention of editing activity has been suggested to correlate with conserved polar contacts between the protein and the hydroxyl at the ribose $2^{\prime}$ position and the need to retain A-form-like helical architecture $69-71,80$. For these apparently conserved positions between Cas 9 and its guide, RNA cannot be readily replaced.

For Cas12a, attempts to fully modify the crRNA guide have proven unsuccessful ${ }^{74,78,81}$. However, the $5^{\prime}$ pseudoknot handle posed the greatest challenge as it appeared to tolerate very little modification to the ribose, suggesting that successful modification of this unique structure would be the major limitation to greater modification of Cas12a guides for therapeutic development. To address this bottleneck, we took a rational, structure-guided approach with respect to predicted 2'-hydoxyl $\left(2^{\prime}-\mathrm{OH}\right)$ polar contacts.

In this work, we utilized chemical modifications that specifically explored ribose and $2^{\prime}-\mathrm{OH}$ properties. We successfully modified the $5^{\prime}$ pseudoknot and obtained very robust gene editing when as few as 6 out of 19 residues remained unmodified. The key positions that remained resistant to modification correlated with predicted $2^{\prime}-\mathrm{OH}$ polar contacts within the Cas12a-crRNA crystal structure ${ }^{20,39}$. The bridging phosphodiester bonds $3^{\prime}$ to these residue positions could further be modified to PS and retain very high cis and trans cleavage activity, as well as gene editing. When crRNAs with chemically modified $5^{\prime}$ pseudoknot handles were screened for trans cleavage activity, some induced 
differential activities like high trans activity with little or no cis dsDNA target cleavage. These results were relegated to the high catalytic turnover of trans activity even when very modest cis cleavage occurred. Together, these results advance efforts toward chemical modification of $A s$ Cas12a guides for therapeutic editing and potential diagnostic applications.

\section{Results}

Probing $2^{\prime}$-OH contacts and A-form preference with $2^{\prime}$-deoxyribose. Acidominococcus species Cas12a (AsCas12a) and a 39nucleotide crRNA were used for this study. The crRNA can be structurally and functionally divided into two domains: a 19nucleotide $5^{\prime}$ handle with a pseudoknot structure that anchors crRNA binding to Cas $12 \mathrm{a}$ and a 20 -nucleotide $3^{\prime}$ guide region that base-pairs to complementary target strand DNA (Fig. 1a). A crystal structure of $A s$ Cas12a has enabled prediction of potentially important polar contacts or hydrogen bonds between AsCas12a and the RNA via $2^{\prime}$-hydroxyl $\left(2^{\prime}-\mathrm{OH}\right)$ groups in the pseudoknot structure, as well as intramolecular 2'-OH RNA-RNA contacts ${ }^{20}$. The importance of RNA A-form helical structure and $2^{\prime}-\mathrm{OH}$ chemistry has been partially investigated for pseudoknot stability and protein interaction ${ }^{85,86}$, but not in the context of CRISPRCas systems. The unique noncanonical nature of the Cas12a $5^{\prime}$ pseudoknot, as well as previous attempts at modification ${ }^{74,78,81}$, suggested that it would be challenging to modify for therapeutic development. To focus on the pseudoknot structure and uncouple it from guide region effects, we maintained an entirely native RNA ribose $\left(2^{\prime}-\mathrm{OH}\right)$ guide region throughout most of this study. This was accomplished through splint ligation of a $5^{\prime}$ phosphorylated guide RNA and chemically modified pseudoknot RNAs using T4 DNA ligase ${ }^{87}$ (Supplementary Fig. 1) or direct solid-phase chemical synthesis of the entire crRNA when modification schemes prevented efficient enzymatic ligation. Enzymatic ligation facilitated more cost-effective and modular production of full-length crRNAs. To investigate the role of the ribose $2^{\prime}-\mathrm{OH}$ and A-form structural preferences in $5^{\prime}$ pseudoknot structure-function for $A s$ Cas12a, we substituted RNA nucleotides with $2^{\prime}$-deoxy (DNA), $2^{\prime}$-fluoro $\left(2^{\prime}\right.$-F), oxepane $(\mathrm{OxN}), 2^{\prime}$-arabino $\left(2^{\prime}\right.$-araOH), and $2^{\prime}$-amino $\left(2^{\prime}-\mathrm{NH}_{2}\right)$ residues (Fig. $\left.1 \mathrm{~b}\right)$.

While DNA nucleotides can probe the importance of $2^{\prime}-\mathrm{OH}$ contacts, they introduce conformational flexibility and can therefore also probe the importance of A-form structural preferences $69,88,89$. Pseudoknots are noncanonical RNA structures that have also been generated with single-stranded DNA $^{90,91}$. Thus, we initially synthesized a crRNA with all nucleotides in the pseudoknot substituted with DNA (cpEGIPD1). No sequence-specific (cis) cleavage activity was observed for this design in vitro, indicating that either $2^{\prime}-\mathrm{OH}$ contacts or A-form helical structure, or both were necessary for Cas12a RNP assembly or enzyme activity (Fig. 2a). Based on an AsCas12a crystal structure ${ }^{20}$, we identified putative critical $2^{\prime}-\mathrm{OH}$ contacts in the pseudoknot structure at positions $-1,-6,-10,-13,-14,-17$, -18 , and -19 . Converting these positions back to RNA, except -19 , (cpEGIP-N1) completely rescued in vitro cleavage activity. These results, along with inspection of the Cas12a crystal structure, suggested to us that the $2^{\prime}-\mathrm{OH}$ at residue - 19 may not be a critical polar contact that impacts activity. Conversely, an all-RNA pseudoknot with the same seven potentially critical $2^{\prime}-\mathrm{OH}$ contact positions (omitting position -19) replaced with DNA (cpEGIP-N2) only provided a quarter of the normal AsCas12a activity. Thus, these results supported the critical role of $2^{\prime}-\mathrm{OH}$ contacts for either pseudoknot structure or protein interaction. However, the observation that cpEGIP-N2 still conferred cleavage activity with no $2^{\prime}-\mathrm{OH}$ groups at the predicted critical positions also suggested that A-form helical structure or $\mathrm{C}^{\prime}$-endo sugar pucker may play an important, albeit potentially lesser role. Indeed, adding additional DNA nucleotides to cpEGIP-N2 to create cpEGIP-A1, which possessed only six RNA residues, abrogated cleavage activity. The activity of cpEGIP-N2, although low, also suggested that pseudoknot $2^{\prime}-\mathrm{OH}$ contacts may not be obligatory for activity.

To further test the role of individual positions and explore RNA-DNA combinations, we synthesized and tested several more crRNAs. Starting with a design that preserved the putative critical 2'$\mathrm{OH}$ contacts and included more RNA residues (cpEGIP-B1), we replaced $-19,-18$, and -17 positions individually (cpEGIP-B1a, $-B 1 b$, and -B1c). None of these substitutions were detrimental to activity (Fig. 2a). We then added more DNA residues, including at positions -14 and -1 . This resulted in substantially reduced activity (cpEGIPB1d). Adding RNA back to positions - 14 and -15 improved activity (cpEGIP-B1e). Placing a DNA nucleotide at the putative critical 2'$\mathrm{OH}$ position -6 , as well as -3 , showed even greater enzyme activity (cpEGIP-B1f), suggesting that -6 is more tolerant of $2^{\prime}-\mathrm{OH}$ loss. Comparing two pseudoknots with the same DNA substitution pattern but with or without DNA at position -1 (cpEGIP-B1g and -B1h) revealed that DNA at -1 was not only well tolerated but also seemed to enhance the activity. These results suggested that no specific critical 2'-OH position was necessarily more important than another and instead that their cumulative loss is additive and negatively impacts enzyme activity.

To test both specific positions and cumulative DNA effects, we systematically walked overlapping blocks of 4-6 DNA nucleotides across the pseudoknot sequence (cpEGIP-E1 through -E5). AsCas12a exhibited high activity with all these crRNAs (Fig. 2a). This result supports the notion that no individual RNA position is essential and high activity can be achieved in vitro with DNA substitutions if sufficient RNA nucleotide content is preserved. Indeed, a new series of crRNAs where we added increasing numbers of DNA residues, including at potentially critical $2^{\prime}-\mathrm{OH}$ contact positions (cpEGIP-F1 through -F6), abrogated AsCas12a activity. These results support the important role of A-form helical preference or reduced flexibility introduced by $\mathrm{C}^{\prime}$-endo sugar pucker.

Previous studies by us and others have determined that specific native $2^{\prime}-\mathrm{OH}$ ribose chemistry (RNA nucleotides) is not necessary for in vitro cleavage but is required for efficient gene editing in cells by CRISPR-Cas9 from S. pyogenes ${ }^{70,71,80}$. Critical $2^{\prime}-\mathrm{OH}$ contact positions lie in the SpCas9 crRNA guide (spacer) seed region and the repeat region proximal to the guide ${ }^{70,71}$. Thus, they are localized in the center of the SpCas9 crRNA. Furthermore, RNA-DNA chimeric Cas9 crRNAs composed of DNA with only RNA at the critical $2^{\prime}-\mathrm{OH}$ positions were highly active in vitro ${ }^{69}$. To determine if a similar phenomenon existed for the $5^{\prime}$ pseudoknot of Cas12a, we selected several RNA-DNA chimeric pseudoknot crRNAs with high activity in vitro and tested their ability to knock out, and therefore edit, an EGFP gene. We started with HEK293T cells stably expressing EGFP 79 and transduced them with a $3 \mathrm{xNLS}-A s$ Cas12a-expressing lentivector followed by selection (Supplementary Fig. 2). crRNAs were lipidtransfected into these custom HEK293T cells constitutively expressing EGFP and AsCas12a. For cell-based editing experiments, $5^{\prime}$ pseudoknots were ligated to a different guide sequence (hence the designation cpEGIPe) designed to target the integrated EGFP gene (Supplementary Fig. 2). Our native all-RNA control routinely generated about $70-80 \%$ knockout of EGFP when measured by flow cytometry 5 days after transfection (Fig. $2 \mathrm{~b}$ and Supplementary Fig. 2d). However, the RNA-DNA chimeric pseudoknots generated little or no editing activity. Notably, cpEGIPe-N1 was inactive despite preserving RNA at the putative critical 2'-OH positions. Only cpEGIPe-B1a provided modest editing (approximately 10\%), perhaps due to better conservation of A-form structure by additional RNA nucleotides. Thus, as had 

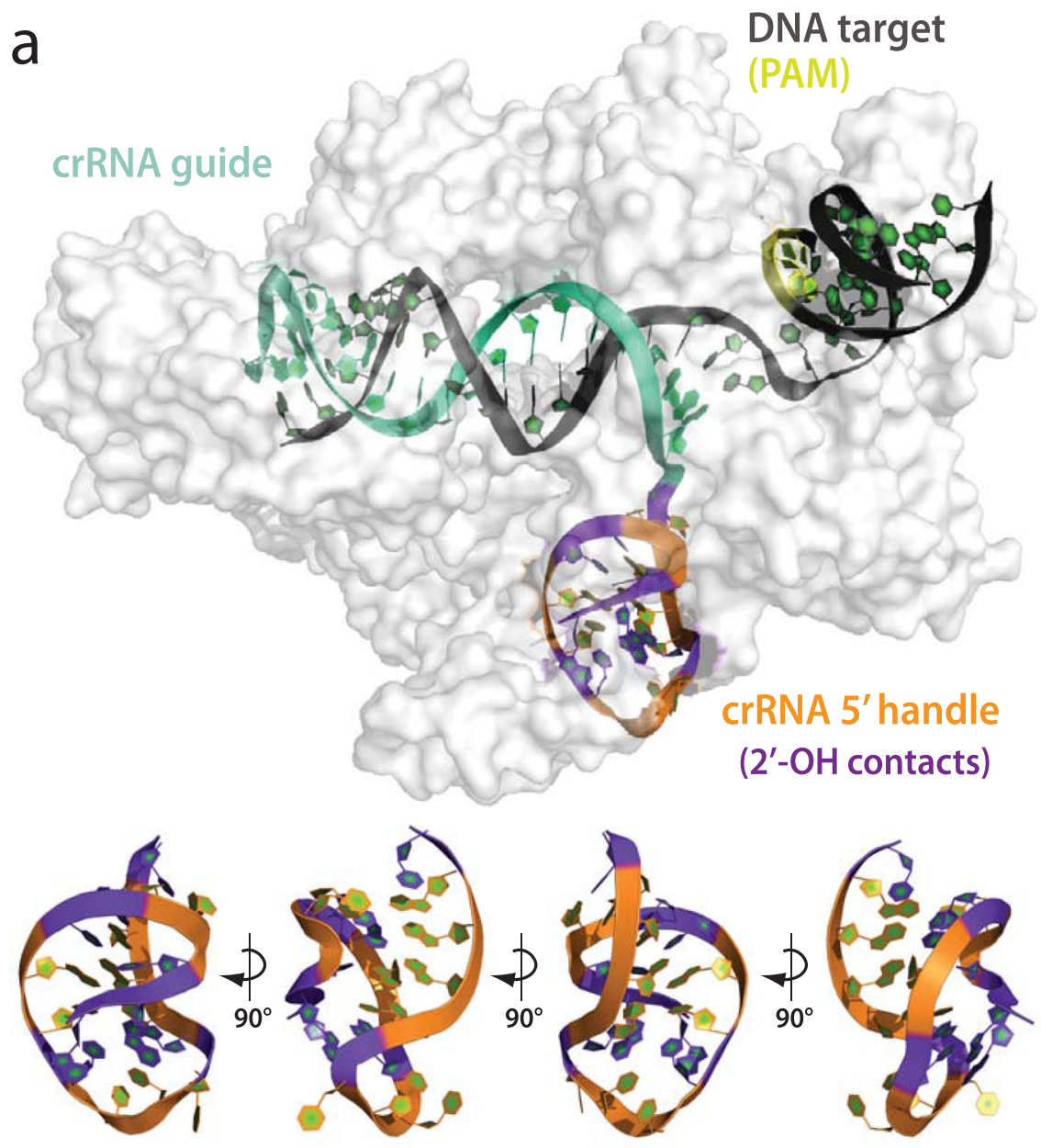

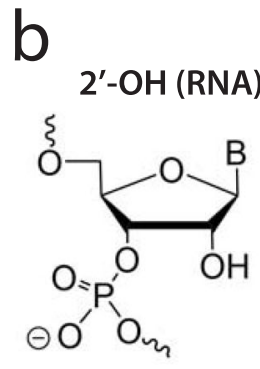<smiles>[Z]OC1OC([B])CC(OP(=O)([O-])O[Na])C1O[Na]</smiles><smiles>[B][C@@H]1O[C@H](COC)[C@@H](F)[C@@H](OP(=O)([O-])O[O-])[C@@H]1F</smiles>

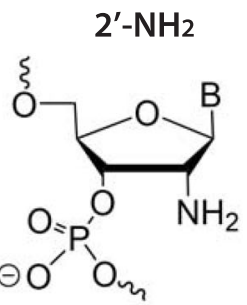

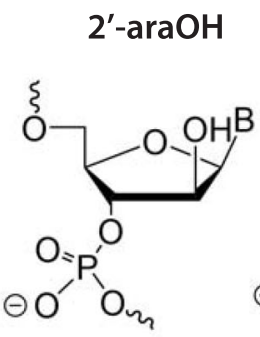<smiles>[3H]C1C[C@@H](O)[C@H](OP(=O)(O)O)[C@@H](O)[C@H](COC)O1</smiles>

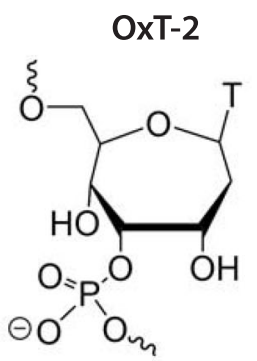<smiles>[3H]C1C[C@H](OP(=O)([O-])O)[C@@H](O)[C@H](O)[C@@H](OC)O1</smiles>

Fig. 1 Structural overview of AsCas12a ribonucleoprotein complex, the crRNA 5 ' pseudoknot, and chemical modifications incorporated into the pseudoknot. a AsCas12a 5' handle pseudoknot structure (PDB ID 5B43). Cas12a protein is rendered as a surface (light gray), DNA target as dark gray, PAM sequence in yellow, crRNA guide in teal, $5^{\prime}$ handle in orange, and $5^{\prime}$ handle nucleotides that make $2^{\prime}-\mathrm{OH}$ polar contacts within the structure in purple. b Chemically modified ribose analogs and substitutes used in this study. 


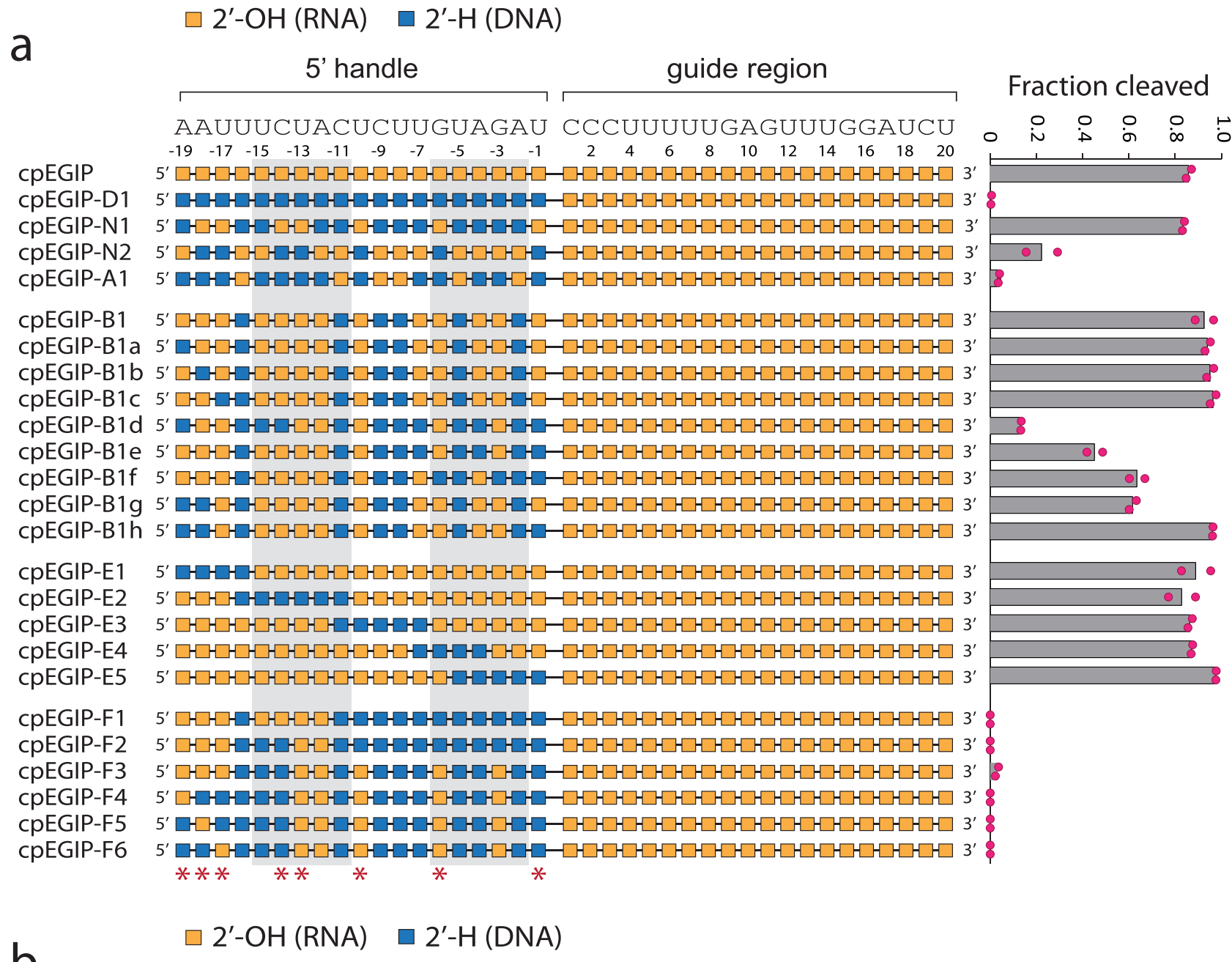

b

\section{5 ' handle}

guide region

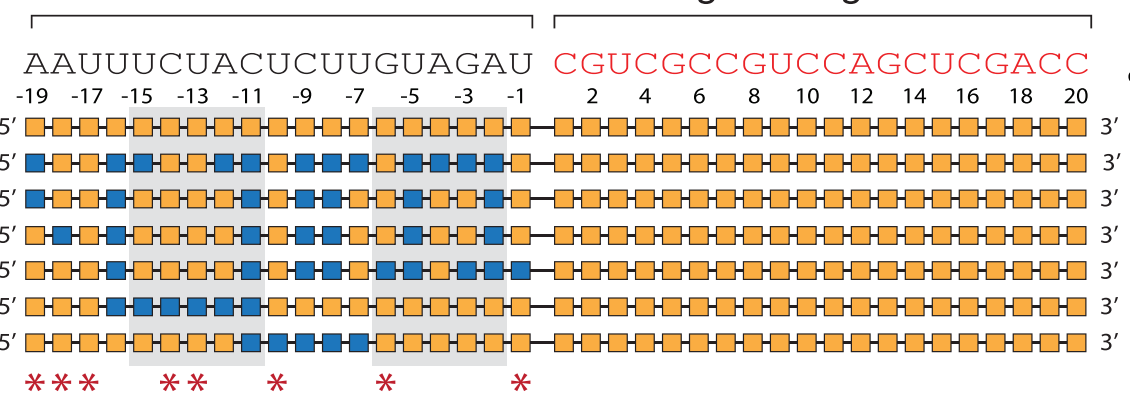

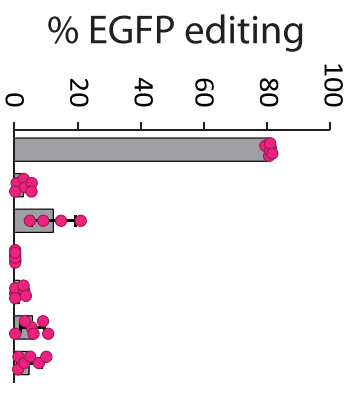

Fig. 2 In vitro cleavage and cell-based editing activity of AsCas12a using crRNA guides modified with 2'-deoxyribose (DNA) nucleotides. DNA modification patterns are illustrated, with corresponding mean in vitro cleavage activity $(n=2)$ (a) or mean cell-based editing activity $(n=6)(\mathbf{b})$ shown to the right. Error bars are standard deviation and replicates are biological. For both panels, nucleotides of the $5^{\prime}$ handle that are predicted from structural data to make polar contacts through 2'-OH hydrogen bonding are indicated with red asterisks below. The base-paired stem nucleotides of the $5^{\prime}$ handle pseudoknot are enclosed with light gray boxes. In vitro cleavage and cell-based editing assays utilize the distinct guide sequences that are shown above each schematic. Source data are provided as a Source Data file.

been observed with SpCas9 crRNAs, retaining $2^{\prime}-\mathrm{OH}$ at potentially critical positions appeared to be necessary but not sufficient to provide gene editing activity ${ }^{70,71}$. These results highlight the more complex nature of editing in cells and support the complementary role of both A-form helical structure and $2^{\prime}$ $\mathrm{OH}$ contacts within the Cas12a crRNA $5^{\prime}$ pseudoknot.

$2^{\prime}$-OH substitutions and RNA mimics in the $5^{\prime}$ pseudoknot. To further investigate the nature of A-form helical preference and ribose chemical compatibility in $5^{\prime}$ pseudoknot structure-activity, we substituted RNA nucleotides with additional modified ribose nucleotides or sugar replacements. The first design was a complete substitution of the pseudoknot with $2^{\prime}$-fluoro-ribose $\left(2^{\prime}-\mathrm{F}\right)$ (cpEGIP-SJ01). This fully modified pseudoknot provided very high cleavage activity, similar to or higher than the native allRNA control (Fig. 3a). The robust activity of this modified pseudoknot indicated that the hydroxyl chemistry at the $2^{\prime}$ position was completely dispensable for intrinsic enzyme activity. Importantly, $2^{\prime}-\mathrm{F}$ is known to stabilize $\mathrm{C}^{\prime}$-endo sugar pucker and 
a

cpEGIP-SJ01
cpEGIP-SJ03
cpEGIP-SJ04
cpEGIP-SJ05
cpEGIP-SJ06
cpEGIP-SJ09
cpEGIP-SJ10
cpEGIP-SJ12
cpEGIP-SJ13
cpEGIP-SJ14
cpEGIP-SJ15
cpEGIP-SJ16
cpEGIP-SJ17

$\square 2^{\prime}-\mathrm{F}$

5 ' handle

\section{Г}

A AUUUCUACUCUUTUAGAU

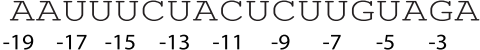

$\begin{array}{lllllllllll}2 & 4 & 6 & 8 & 10 & 12 & 14 & 16 & 18 & 20\end{array}$

$5^{\prime}$ 맘ㅁㅁㅁㅁㅁㅁㅁㅁㅁㅁㅁㅁㅁㅁㅁㄷㅁㅁㅁㅁㅁㅁㅁㅁㅁㅁㅁㅁㅁㅁㅁㅁㅁㅁ $3^{\prime \prime}$

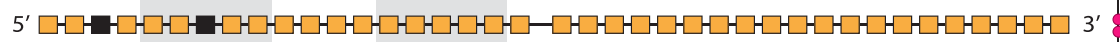

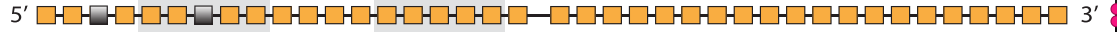

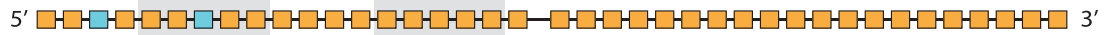

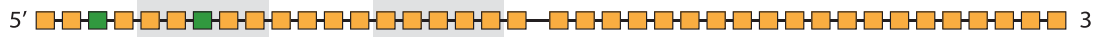

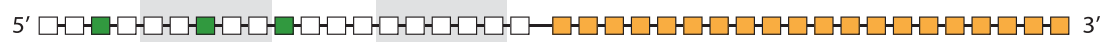

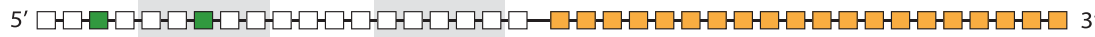

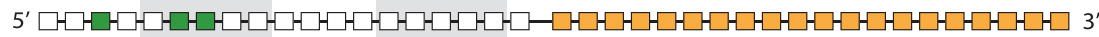

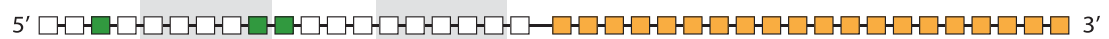

5' 맘ㅁㅁㅁㅁㅁㅁㅁㅁㅁㅁㅁㅁㅁㅁㅁㅁㅁㅁㅁㅁㅁㅁㅁ 3'

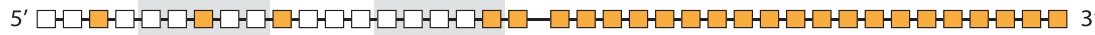

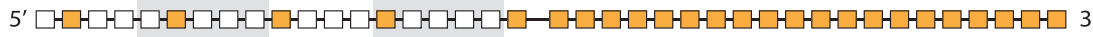

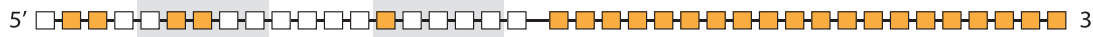

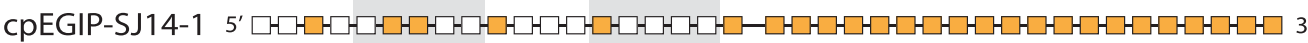

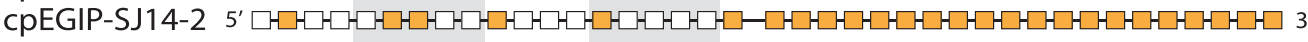

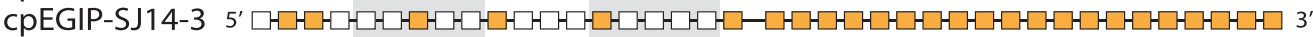

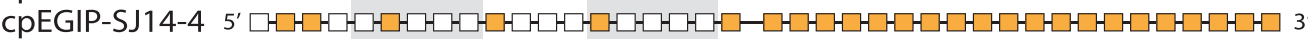

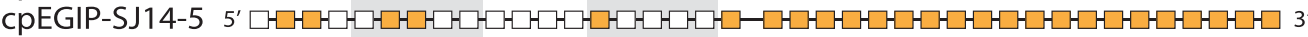

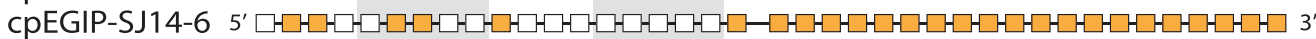

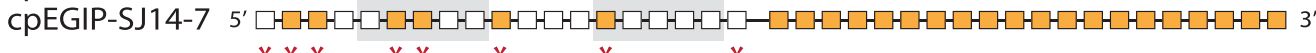
*** * * * * * *
b
$\square 2^{\prime}-\mathrm{OH}$
$\square 2^{\prime}-\mathrm{F}$

\section{2'-araOH}
5' handle

guide region

\begin{tabular}{|c|c|}
\hline & . \\
\hline $7 \mathrm{THTTCT}$ & GUCCAGCUCGA \\
\hline $\begin{array}{ll}-5 & -3\end{array}$ & $\begin{array}{lllllll}8 & 10 & 12 & 14 & 16 & 18 & 20\end{array}$ \\
\hline
\end{tabular}

cPEGIPe

CPEGIPe-SJ01

cpEGIPe-SJ10

CpEGIPe-SJ14

CPEGIPe-SJ15

cpEGIPe-SJ16

CPEGIPe-SJ17

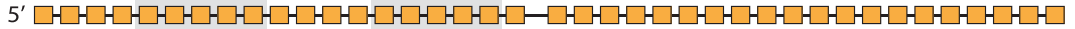

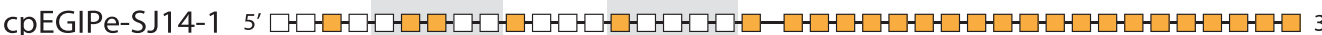

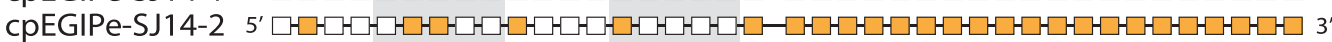

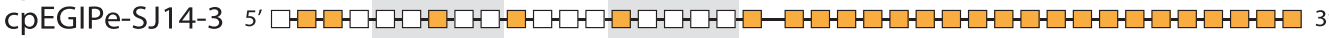

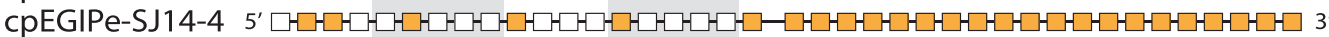

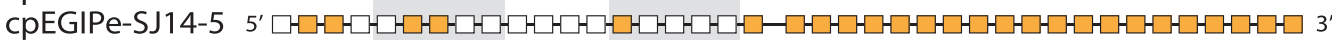

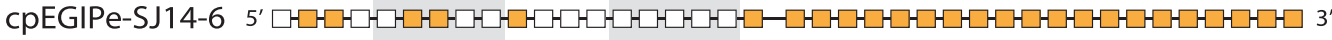

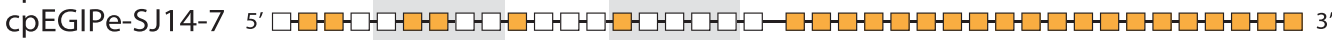

$$
\text { *** * * * * * }
$$

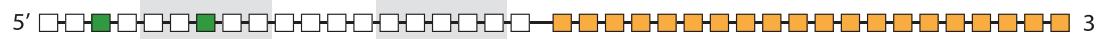

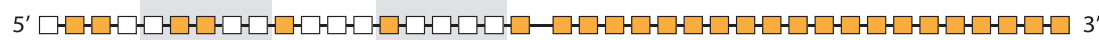

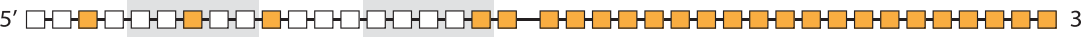

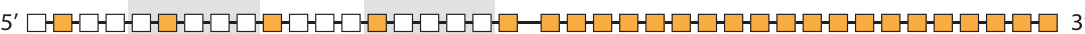

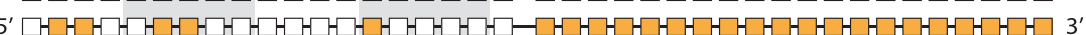

Fig. 3 In vitro cleavage and cell-based editing activity of AsCas12a using crRNA guides modified with 2'-F, oxepane nucleotides, and 2'-araOH. Chemical modification patterns are illustrated with corresponding mean in vitro cleavage activity $(n=2)(\mathbf{a})$ or mean cell-based editing activity $(n=6)(\mathbf{b})$ shown to the right. Error bars are standard deviation and replicates are biological. For both panels, nucleotides of the $5^{\prime}$ handle that are predicted from structural data to make polar contacts through $2^{\prime}-\mathrm{OH}$ hydrogen bonding are indicated with red asterisks below. The base-paired stem nucleotides of the $5^{\prime}$ handle pseudoknot are enclosed with light gray boxes. In vitro cleavage and cell-based editing assays utilize the distinct guide sequences that are shown above each schematic. Source data are provided as a Source Data file.

strongly favor A-form helical structure ${ }^{92,93}$. However, when tested for cell-based editing activity cpEGIP-SJ01 was completely inactive (Fig. 3b). 2'-F nucleotides are potentially able to accept hydrogen bonds but not donate them, making them an incomplete replacement for RNA where 2 '-OH contacts may be critical. While the combination of stable structure, mimicry of RNA properties, and maintenance of sufficient $2^{\prime}$ contacts $^{93}$ have enabled $2^{\prime}-\mathrm{F}$ to substitute for $2^{\prime}-\mathrm{OH}$ in vitro, cell-based editing is apparently more sensitive and likely requires retention of specific $2^{\prime}-\mathrm{OH}$ contacts.

To specifically investigate positions with predicted $2^{\prime}-\mathrm{OH}$ contacts, we synthesized crRNAs with RNA or $2^{\prime}$-F pseudoknots containing oxepane nucleic acid $(\mathrm{OxN})$ and $2^{\prime}$-araOH in putative critical $2^{\prime}-\mathrm{OH}$ positions. OxN is a seven-membered ring structure that can be synthesized with multiple hydroxyl groups and a phosphodiester linkage at different ring positions ${ }^{94}$. We 
synthesized thymidine (OxT) nucleotide replacements with the phosphodiester linkage at three different positions, designated OxT-1, -2, and -3 . These were then incorporated into the pseudoknot sequence at putative critical $2^{\prime}-\mathrm{OH}$ positions -13 and -17 during solid-phase synthesis. The incorporation of OxN (cpEGIP-SJ03, -SJ04, and -SJ05) completely abrogated AsCas12a activity, indicating that certain OxN properties, such as the bulkiness of the seven-membered ring or conformational restraints, may simply be incompatible despite providing hydroxyl groups at various positions (Fig. 3a). Based on these results we chose to not proceed further with OxN.

Arabinose nucleotides $\left(2^{\prime}\right.$-araOH) are stereoisomers of RNA that place the hydroxyl group at the $2^{\prime}$ position on the opposite face of the ribose ring. While arabinonucleosides retain a $2^{\prime}-\mathrm{OH}$ group, the "up" orientation steers the arabinose sugar to adopt a $\mathrm{C}^{\prime}$-endo (DNA-like) conformation ${ }^{95}$. When placing $2^{\prime}$-araOH at -13 and -17 positions (cpEGIP-SJ06), some enzyme activity was retained. We therefore chose to explore more $2^{\prime}$-araOH substitutions but in the background of a $2^{\prime}-\mathrm{F}$ pseudoknot, reasoning that the strong A-form preference of $2^{\prime}$-F might offset structural preferences of $2^{\prime}$-araOH. Incorporating more than two $2^{\prime}$-araOH (cpEGIP-SJ09, -SJ12, and -SJ13) severely reduced or eliminated enzyme activity (Fig. 3a). Only cpEGIP-SJ10, with 2'araOH at positions -13 and -17 , supported high in vitro AsCas12a cleavage activity. These results suggest that $2^{\prime}-\mathrm{F}$ may be successfully combined with other modifications that would otherwise be too detrimental to support high activity. Despite the promising in vitro activity of cpEGIP-SJ10, it provided no editing in cells (Fig. 3b).

$2^{\prime}$-Fluoro, $2^{\prime}-\mathrm{OH}$, and $2^{\prime}$-amino combinations in the $5^{\prime}$ pseudoknot. The high in vitro activity of $2^{\prime}-\mathrm{F}$ and its ability to counteract detrimental effects of $2^{\prime}$-araOH incorporation prompted us to create pseudoknots with a combination of $2^{\prime}-\mathrm{F}$ and RNA nucleotides that might improve cell-based editing. We made an all-2'-F pseudoknot with RNA nucleotides at putative critical 2'-OH positions (except -19). This design, cpEGIP-SJ14, supported very high in vitro cleavage (cis) activity (Fig. 3a). Importantly, when we transfected this same design (cpEGIPeSJ14) into HEK293T cells stably expressing EGFP and AsCas12a, we observed robust editing of EGFP at levels as high or greater than our native RNA control cpEGIPe (Fig. 3b). Other designs that further reduced the number of RNA nucleotides to 5 and incorporated 2'-F at putative critical positions (cpEGIP-SJ15, -16, and -17) did not affect in vitro cleavage. However, the analogous designs targeting cellular EGFP showed varying degrees of reduced editing efficiency (Fig. 3b). This result supports the critical role of the putative $2^{\prime}-\mathrm{OH}$ positions and the value of modifications that retain strong A-form-like helical structure for high editing efficiency.

Different degrees of editing among RNA and 2'-F combinations suggested that some $2^{\prime}-\mathrm{OH}$ interactions were more critical than others, but clear patterns were difficult to discern. Therefore, we created a new series of cpEGIP-SJ14 designs where each of the seven remaining RNA nucleotide were individually substituted with a $2^{\prime}$-F residue. While all designs were highly active in vitro (Fig. 3a), clearer trends were observed for cell-based editing. Placing $2^{\prime}-\mathrm{F}$ at -18 and -17 in the SJ14 design (cpEGIPe-SJ14-1 and -SJ14-2) was very detrimental to editing, reducing it to below 10\% (Fig. 3b). Positions $-14,-13$, and -10 could be individually replaced by $2^{\prime}-\mathrm{F}$ (cpEGIPe-SJ14-3, -SJ14-4, and -SJ14-5) with only mild reductions in editing compared to the RNA control. Surprisingly, replacement at position -6 (cpEGIPe-SJ14-6) resulted in very robust editing of $\sim 90 \%$, suggesting that this position is less sensitive and can be replaced readily with $2^{\prime}-\mathrm{F}$. This result reinforced our initial finding with DNA replacements that suggested position -6 does not depend heavily on 2-OH contacts or chemistry (Fig. 2a). Finally, replacing position -1 with $2^{\prime}-\mathrm{F}$ created strong reductions in gene editing activity. Walking $2^{\prime}-\mathrm{F}$ through the remaining putative $2^{\prime}-\mathrm{OH}$ positions identified a crRNA with a pseudoknot having only six RNA residues out of 19 (cpEGIPe-SJ14-6) that produced AsCas12a editing activity as high or greater than a native crRNA. It also identified higher sensitivity to $2^{\prime}$-F substitution at the terminal ends of the pseudoknot sequence.

The positive performance of $2^{\prime}$-F but generally poor performance of $2^{\prime}-\mathrm{araOH}$ and $\mathrm{OxN}$ indicated that smaller structural perturbations of the ribose and the ability to provide hydrogenbonding potential at the $2^{\prime}$ position were desirable. To determine if further RNA reduction at putative critical $2^{\prime}-\mathrm{OH}$ positions might be possible, we identified $2^{\prime}$-amino $\left(2^{\prime}-\mathrm{NH}_{2}\right)$ as a potentially promising modification. The relatively small amino moiety and its ability to both donate and accept hydrogen bonds made it an attractive replacement for RNA. In addition, the pKa of the $2^{\prime}$-amino should be 6.2, as determined by Eckstein and colleagues $^{96}$, making the major species non-protonated at physiological pH. $2^{\prime}-\mathrm{NH}_{2}$ nucleotides have been used for other technologies, like aptamer and ribozyme development ${ }^{97-99}$, but have not been applied to CRISPR guide RNA modification previously. Starting with an all-2'-F pseudoknot, we sought to replace the putative critical positions individually with $2^{\prime}-\mathrm{NH}_{2}$ to ensure that in vitro activity was not affected. Because $2^{\prime}-\mathrm{NH}_{2}$ is an uncommon modification and only pyrimidine monomers were commercially available, we did not substitute positions -18 and -6 . All the crRNAs containing a $2^{\prime}-\mathrm{NH}_{2}$ substitution in the $2^{\prime}-\mathrm{F}$ pseudoknot (cpEGIP-LA-N14-1 through -LA-N14-5) were highly active in vitro (Fig. $4 \mathrm{a}$ ), indicating no negative impact on intrinsic enzyme activity. However, none of these designs targeting EGFP in cells showed significant gene editing activity (Fig. 4b).

To begin with modified pseudoknots more likely to generate gene editing activity, we created the equivalent of cpEGIP-SJ14 ( $\sim 80 \%$ editing) but walked a $2^{\prime}-\mathrm{NH}_{2}$ through each remaining native RNA nucleotide position (except -18 and -6 ). These new designs, cpEGIP-LA-N14-6 through -LA-N14-10, all exhibited robust in vitro cleavage activity (Fig. 4a). When targeting the stably expressed EGFP gene in cells, these designs presented varying degrees of gene editing. Substitution at position -17 with $2^{\prime}-\mathrm{NH}_{2}$ abrogated gene editing activity (cpEGIPe-LA-N14-6). Substitution at positions - 13 and - 1 resulted in approximately $30 \%$ gene editing (cpEGIPe-LA-N14-8 and -LA-N14-10). In contrast, substitutions with $2^{\prime}-\mathrm{NH}_{2}$ at positions -10 and -14 (cpEGIPe-LAN14-9 and -LA-N14-7) provided $\sim 60-70 \%$ editing, which is substantial considering that the native RNA pseudoknot typically provides $80 \%$ editing. Interestingly, substitution at position -14 resulted in similar activities whether 2'-F (cpEGIPe-SJ14-3) or 2'$\mathrm{NH}_{2}$ (cpEGIPe-LA-N14-7) were used, suggesting relatively flexible accommodation at this position. $2^{\prime}-\mathrm{NH}_{2}$ was not suitable for replacing RNA at positions -17 or -1 , which was also the case with 2'-F (cpEGIPe-SJ14-2 and cpEGIPe-SJ14-7). However, position -13 was differentially affected, with $2^{\prime}-\mathrm{F}$ being very well-tolerated (cpEGIPe-SJ14-4), whereas $2^{\prime}-\mathrm{NH}_{2}$ was poorly tolerated (cpEGIPe-LA-N14-8). Amines do not form hydrogen bonds as stably as alcohols and are bulkier at the $2^{\prime}$ position than $\mathrm{OH}$ and $\mathrm{F}$, which may explain differential activity effects.

Trans cleavage activity of modified Cas12a crRNAs. Differences in sequence-specific cis cleavage in vitro versus cell-based gene editing prompted us to investigate whether chemically modified $5^{\prime}$ pseudoknots impacted non-sequence-specific trans cleavage activity. We employed a fluorescence-based assay similar to that originally reported by Chen and coworkers ${ }^{41}$ (Supplementary 


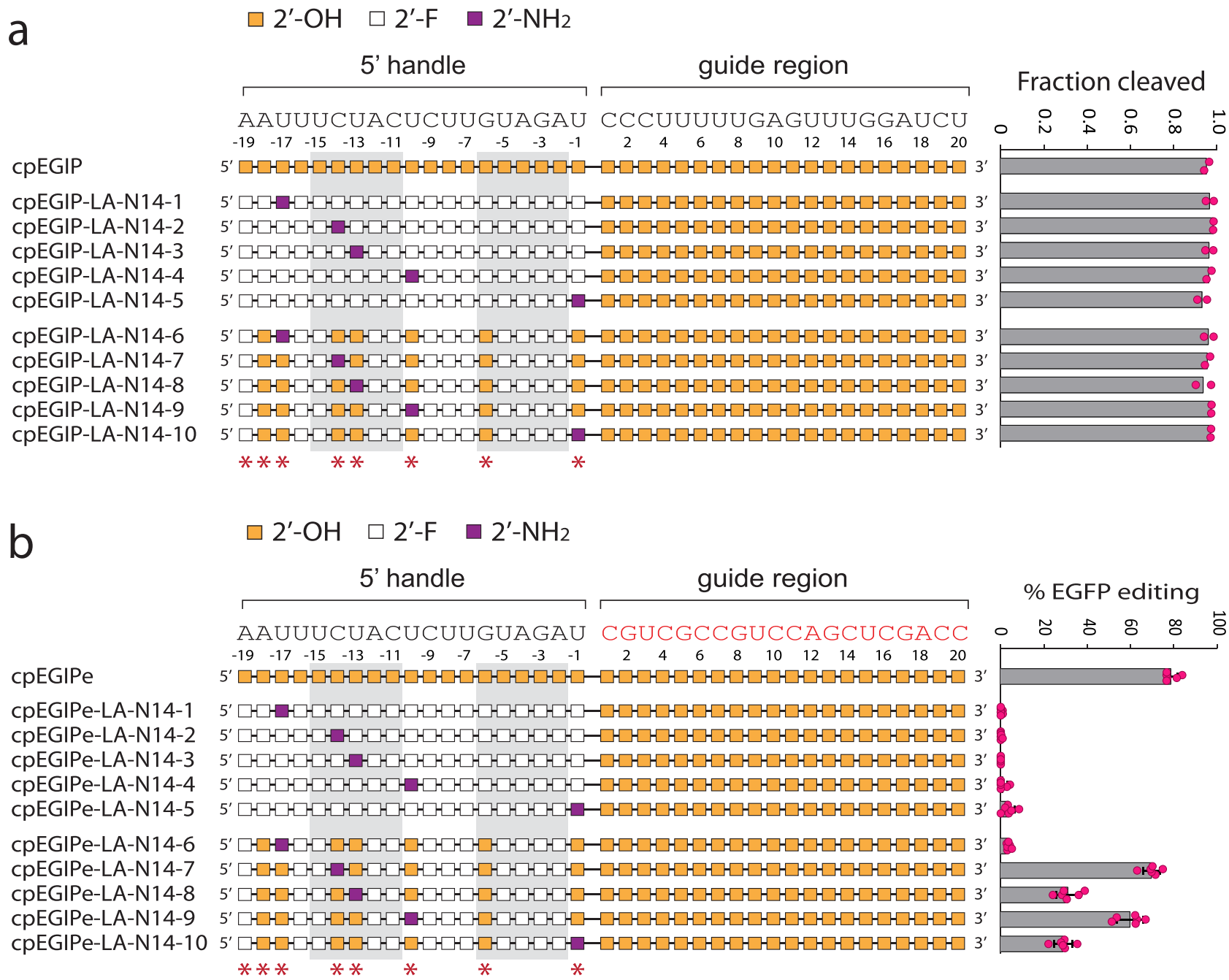

Fig. 4 In vitro cleavage and cell-based editing activity of AsCas12a using crRNA guides modified with 2'-F and 2'-NH $\mathbf{~}_{\mathbf{2}}$ combinations. Chemical modification patterns are illustrated with corresponding mean in vitro cleavage activity $(n=2)$ (a) or mean cell-based editing activity $(n=6)(\mathbf{b})$ shown to the right. Error bars are standard deviation and replicates are biological. For both panels, nucleotides of the $5^{\prime}$ handle that are predicted from structural data to make polar contacts through 2'-OH hydrogen bonding are indicated with red asterisks below. The base-paired stem nucleotides of the $5^{\prime}$ handle pseudoknot are enclosed with light gray boxes. In vitro cleavage and cell-based editing assays utilize the distinct guide sequences that are shown above each schematic. Source data are provided as a Source Data file.

Fig. 3a). We systematically screened all crRNAs with modified pseudoknots and plotted their maximum trans activity after $1 \mathrm{~h}$ of reaction time. We found that levels of trans and cis activity closely mirrored one another for most crRNAs (Fig. 5). This would be expected since trans cleavage activity relies on the same active site as cis cleavage activity ${ }^{45,46}$. However, a few crRNAs with modified $5^{\prime}$ pseudoknots generated differential cis versus trans activity. For example, cpEGIP-B1d and cpEGIP-F6 both had little or no cis activity but exhibited about $50 \%$ trans activity (Fig. 5a). However, after incubation for $2 \mathrm{~h}$, both reached similar activity levels as the all-RNA control crRNA (Supplementary Fig. 3b). In contrast, cpEGIP-SJ10 and cpEGIP-LA-N14-1 both exhibited very high cis cleavage but low trans cleavage activity $(\sim 10 \%)$ (Fig. $5 \mathrm{~b}, \mathrm{c}$ ). Incubation for over $2 \mathrm{~h}$ resulted in no additional trans cleavage for cpEGIP-SJ10 and less than $40 \%$ for -LA-N14-1 (Supplementary Fig. 3b).

To determine whether the apparent differential cis-trans activity was guide-sequence dependent, we ligated the modified $5^{\prime}$ pseudoknot to the cpEGIPe guide sequence used for editing in cells. With this guide sequence, both cpEGIPe-B1d and cpEGIPeF6 still produced markedly low cis but about 50\% trans activity
(Fig. 5d), which reached full trans activity after an additional 60 min of incubation (Supplementary Fig. 3c). In addition, cpEGIPe-F1 still produced almost no cis activity but now generated very high trans activity. Two modified crRNAs that had previously shown high cis but low trans activity, cpEGIPeSJ10 and cpEGIPe-LA-N14-1, now exhibited similar cis-trans activity profiles. One possible explanation for these results is that chemical modifications may enable conformational changes to Cas12a that allow trans cleavage without target binding. However, trans cleavage assays lacking target DNA for several crRNAs did not produce any significant trans activity (Supplementary Fig. 4a). An alternative explanation for trans activity with little or no cis activity could potentially be "nicking" of one of the target DNA duplex strands. This could conceivably activate Cas 12 a trans activity but not be readily observed in typical cis cleavage assays, which use non-denaturing gel electrophoresis. Therefore, we individually $5^{\prime}$ radiolabeled non-target (sense) and target (antisense) strands and performed trans cleavage assays on duplexes using cpEGIPe and cpEGIPe-B1d, -F1, -F6, and -SJ14-7 crRNAs, followed by resolution of reaction products on denaturing polyacrylamide gels (Supplementary Fig. 4b). The 

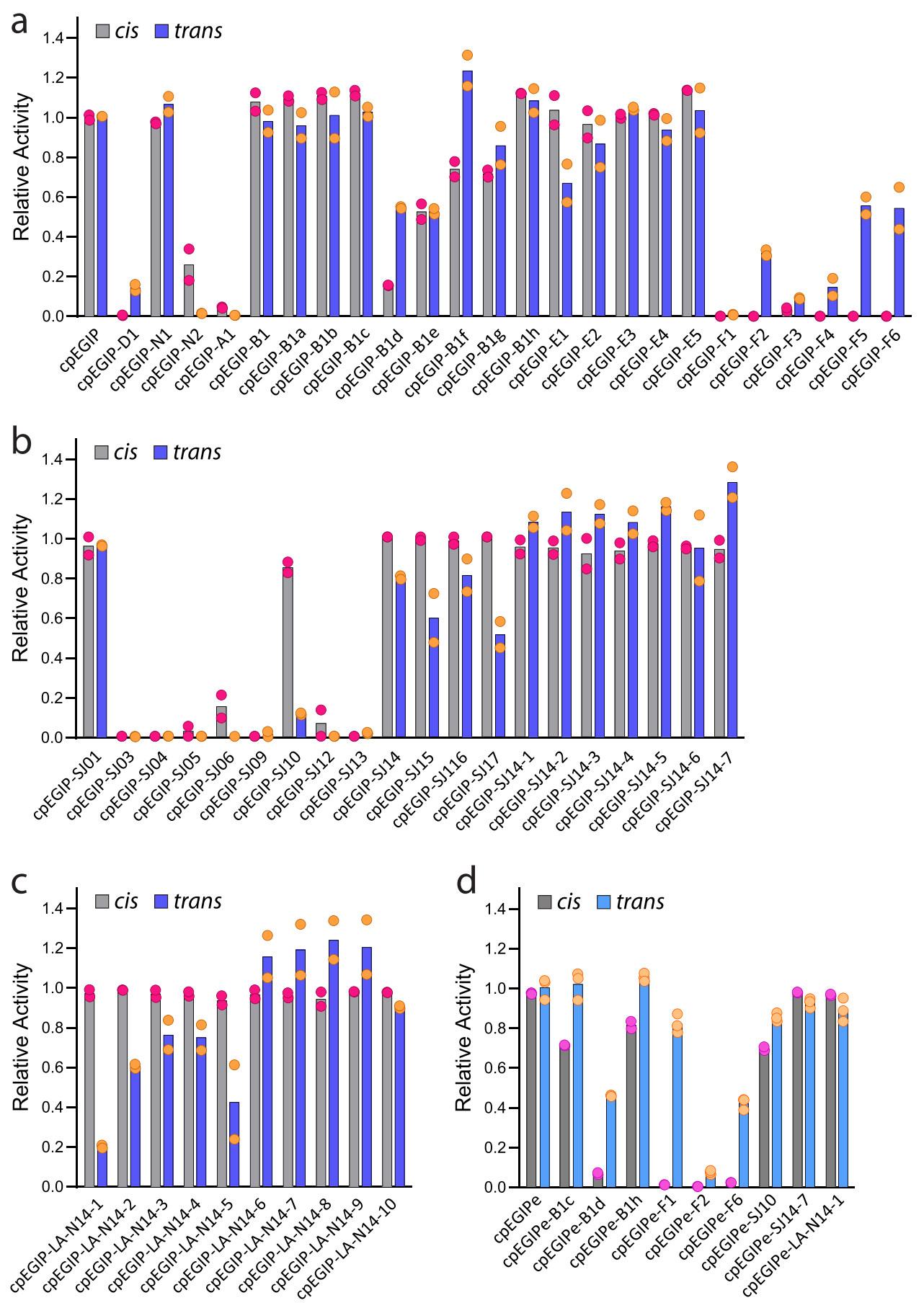

Fig. 5 Chemical modification of the AsCas12a crRNA $5^{\prime}$ handle modulates non-sequence-specific trans ssDNase activity. a-d Comparing dsDNA sequence-specific cis and ssDNA non-specific trans activity of AsCas12a with crRNAs containing modified 5' handles. Error bars are standard deviation and replicates are biological. The guide sequence used in panels (a-c) (cpEGIP) is designed to target an EGFP-containing plasmid for in vitro cis cleavage while the guide used in panel (d) (cpEGIPe) is designed to target a chromosomally integrated EGFP gene for cell-based editing. For all samples, two separate replicates were performed $(n=2)$. Source data are provided as a Source Data file.

cleavage of individual duplex strands very closely matched that of our previous cis assays, indicating that differential strand nicking was not a likely explanation for high trans but low cis activity. To further investigate the mechanism of apparent differential cis-trans activity, we generated hybrid dsDNA targets that incorporated a partially PS-modified non-target (sense) or target (antisense) strand (Supplementary Fig. 5a) ${ }^{45}$. The PS-modified target strand was observed to consistently reduce trans activity by about $60 \%$ for both native and chemically modified pseudoknotcontaining crRNAs while a PS-modified non-target strand had little or no effect (Supplementary Fig. 5b). These results are consistent with trans activity first requiring cis cleavage of the target strand ${ }^{45}$. Thus, we conclude that high catalytic turnover of trans activity ${ }^{41}$ versus the low turnover of cis activity is the most likely explanation for apparent differential cis-trans activity. The modified crRNAs that displayed differential cis-trans activity likely fell into an activity regime that made this effect easily observable. Nonetheless, chimeric RNA-DNA pseudoknots did not appear to change the intrinsic enzyme mechanism of trans cleavage (Supplementary Fig. 5b). 


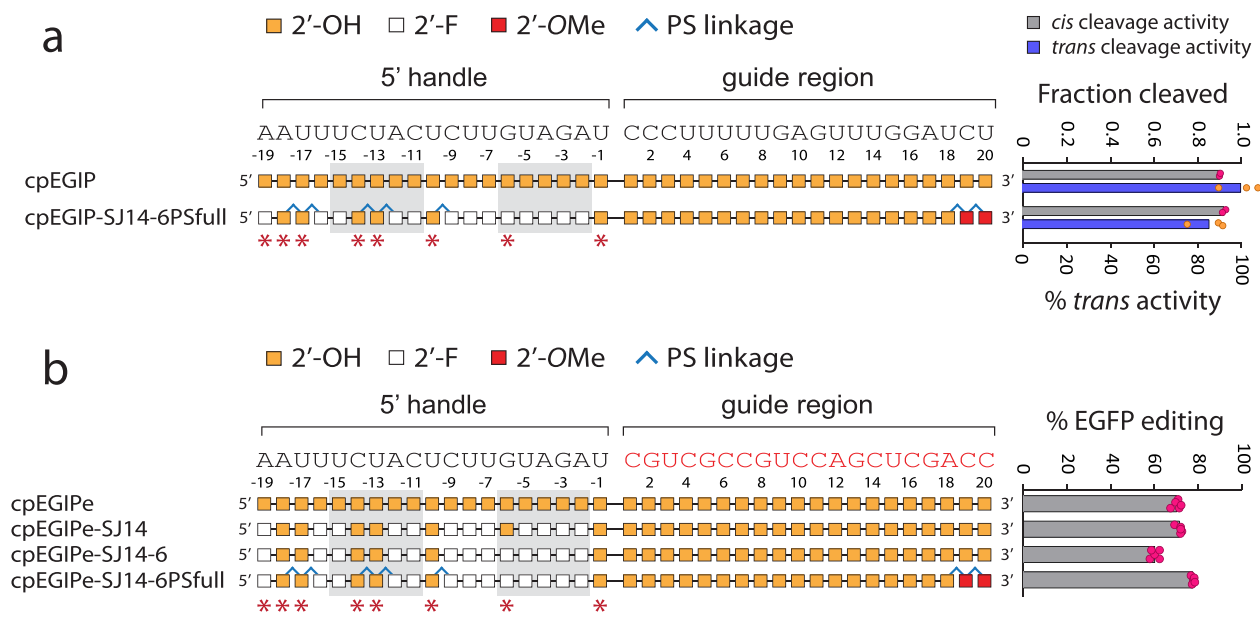

Fig. 6 In vitro cis and trans cleavage activity and cell-based editing of AsCas12a using a heavily modified pseudoknot crRNA guide. Chemical

modification patterns are illustrated with corresponding mean in vitro cis and trans activity $(n=2)(\mathbf{a})$ or mean cell-based editing $(n=5)(\mathbf{b})$ shown to the right. Error bars are standard deviation and replicates are biological. Nucleotides of the $5^{\prime}$ handle that are predicted from structural data to make polar contacts through 2'-OH hydrogen bonding are indicated with red asterisks below. The base-paired stem nucleotides of the 5' handle pseudoknot are enclosed with light gray boxes. In vitro cleavage and cell-based editing assays utilize the distinct guide sequences that are shown above each schematic. Source data are provided as a Source Data file.

The crRNA possessing the maximum number of modifications and exhibiting little or no loss in editing was cpEGIPe-SJ14-6. However, this modification scheme retained six RNA residues, which may be subject to RNase attack in therapeutic contexts. Thus, to further stabilize these final positions, we replaced the phosphodiester linkage $3^{\prime}$ to each remaining RNA residue with PS linkages. This newly modified pseudoknot was ligated to the cpEGIPe guide possessing two terminal $3^{\prime}$ PS linkages and $2^{\prime}-O-$ methyl nucleotides to further stabilize against $3^{\prime}$ exonucleases. This new modified crRNA, cpEGIPe-SJ14-6PSfull, exhibited full cis, trans and editing activities (Fig. 6). Thus, AsCas12a crRNAs can be generated with heavily modified pseudoknots that should protect every nucleotide from nucleases and retain robust editing activity. To test the nuclease resistance of several pseudoknots and ligated crRNAs, we also performed serum stability assays (Supplementary Fig. 6a). Native crRNA (cpEGIPe) and an RNA-DNA chimeric pseudoknot (cpEGIPe-F6) were completely digested under the conditions tested. The cpEGIPe-SJ01 pseudoknot, composed entirely of $2^{\prime}$-F, was preserved while the cpEGIPe-SJ14 pseudoknot, containing $2^{\prime}$-F and seven RNA residues, was significantly degraded. To test the effect of additional PS modifications, full-length crRNAs were generated by enzymatic ligation. Addition of two terminal PS modifications to the SJ14-6 design (cpEGIPe-SJ14-6PS) improved stability of the modified $5^{\prime}$-pseudoknot slightly (lanes 8 and 10). In contrast, the $5^{\prime}$ pseudoknot of cpEGIP-SJ14-6PSfull was well preserved against degradation (lane 12). These results confirm that select $2^{\prime}$ $\mathrm{F}$ and PS modification of the $5^{\prime}$ pseudoknot can stabilize against common serum nucleases.

To determine whether pseudoknot modifications may be impacting enzyme activity through large conformational effects, we performed limited trypsin hydrolysis on AsCas12a RNP complexes (Supplementary Fig. 6b). AsCas12a by itself, assembled as a native RNP with cpEGIPe, or further bound to its cognate dsDNA target revealed unique limited trypsin digestion patterns. However, no difference in the trypsin digestion pattern was observed when comparing native RNP complexes with RNPs assembled from heavily modified pseudoknot guides (cpEGIPeSJ14-6 and cpEGIPe-SJ14-6PSfull) previously shown to possess robust in vitro cleavage and editing activity (Figs. 3 and 6). Further addition of a cognate dsDNA target to modified RNP complexes also showed no differences when compared to the native RNP complex with bound target. Thus, crRNAs with modified pseudoknots that confer high editing activity do not perturb gross AsCas12a conformation.

\section{Discussion}

Chemical modification of RNA is a method to probe the role of functional groups in RNA and RNP structure and biochemical activity ${ }^{71,100}$. It is also a critical step in converting RNA into drug-like molecules ${ }^{66}$. In this study, we have used chemical modification to investigate the necessity of the ribose $2^{\prime}-\mathrm{OH}$ group in the AsCas12a crRNA $5^{\prime}$ pseudoknot structure for enzyme activity. The primary question was whether the pseudoknot structure was too complex or sensitive for efficient chemical modification, which previous studies had suggested ${ }^{74,78,81}$. The rationale for our approach is the high likelihood that therapeutic applications of Cas12a that require direct delivery of the crRNA will also require heavy or complete chemical modification, as is the case for siRNAs. In particular, the lability of RNA is directly linked to the presence of a $2^{\prime}-\mathrm{OH}$ that can help catalyze intramolecular phosphodiester bond self-cleavage or attract ribonucleases ${ }^{101}$. The $5^{\prime}$ pseudoknot anchors crRNA binding to Cas12a and is highly conserved across the CRISPR-Cas12a family $16,39,102$. The conservation of pseudoknot sequence and structure suggests that the $5^{\prime}$ pseudoknot architecture is finetuned for Cas12a function and cannot be easily perturbed 16,102 . However, if successful chemical modification schemes can be identified, they should translate to other Cas12a proteins if RNAprotein contacts are preserved, which appears to be the case from recent structural investigations $20,39,103$.

Here we focused primarily on $2^{\prime}$-deoxyribose, or DNA, and $2^{\prime}$ $\mathrm{F}$ as substitutions and also included modifications designed to potentially retain hydrogen bonding between pseudoknot nucleotides or with AsCas12a protein. We have demonstrated that as many as 13 out of 19 nucleotides can be converted to $2^{\prime}$-F with no impact on efficiency of EGFP editing in human cells. Attempting to systematically modify remaining putative critical $2^{\prime}-\mathrm{OH}$ positions with $2^{\prime}-\mathrm{F}, \mathrm{OxN}, 2^{\prime}$-araOH, and $2^{\prime}-\mathrm{NH}_{2}$ resulted in moderate success. However, it was clear that a few positions are resistant to modification and seem to rely heavily on the $2^{\prime}-\mathrm{OH}$ 
group. For example, our results suggested that the more centrally positioned $2^{\prime}-\mathrm{OH}$ contacts, especially at residues $-14,-10$, and -6 , were more tolerant to substitution while terminal residues $(-18$, -17 , and -1$)$ were less tolerant. Although $\mathrm{OxN}$ and $2^{\prime}$-araOH were not sufficient substitutes for ribose, it appears that a few $2^{\prime}-\mathrm{NH}_{2}$ and $2^{\prime}$-deoxy could be incorporated in the context of $2^{\prime}$-F. Positions most amenable to these modifications are position -19 and more central positions like -6 . In summary, removal of all but a handful of ribose sugars is achievable in the $5^{\prime}$ pseudoknot structure of $A s$ Cas12a crRNAs while still maintaining very high gene editing activity in cells. In addition, selective placement of PS linkages $3^{\prime}$ to remaining RNA nucleotides further stabilized the RNA while not compromising editing activity. A similar approach succeeded in stabilizing remaining RNA positions in a Cas9 guide RNA, although editing in the final heavily modified RNA guide was significantly reduced ${ }^{70}$. Previously, full PS modification of the native $5^{\prime}$ pseudoknot of the AsCas12a crRNA resulted in a substantial loss of editing in cells ${ }^{81}$.

Previous attempts to modify the $5^{\prime}$ pseudoknot sequence replaced entire blocks of contiguous nucleotides, several at a time, with DNA, 2'-F, or $2^{\prime}-O$-methyl ${ }^{74,78}$. This approach is typically suitable for oligonucleotides that function almost exclusively through Watson-Crick base-pairing, such as ASOs or siRNAs ${ }^{66,104}$. However, it resulted in no editing activity, likely due to loss of critical 2 '-OH contacts. Overall, these results reinforce the need to incorporate molecular and structural data into modification design and the value of nucleotide analogs that can mimic or substitute the properties of native ribose.

Ultimately, complete ribose chemical modification of the entire crRNA would be desirable for precise control over therapeutic development. Attempts to modify the guide region of Cas12a crRNAs explored 2'-O-methyl, 2'-F, DNA, LNA, UNA, and PS linkages ${ }^{74,81}$. The general conclusion from these studies was that modification at or near the $3^{\prime}$ end of the guide region is relatively well-tolerated for most modifications. However, modification at the $5^{\prime}$ end of the guide, and therefore within or near the "seed" region, were very poorly tolerated. A notable feature of the seed region of both Cas12a and Cas9 CRISPR systems is the concentrated presence of conserved $2^{\prime}-\mathrm{OH}$ contacts with the Cas protein 12,20 . In addition, the ability for Cas9 to recognize and bind DNA targets, as well as catalyze cleavage, has been shown to be dependent on the formation of an A-form-like duplex between the guide and target DNA ${ }^{69,71}$. Together, the $2^{\prime}-\mathrm{OH}$ and A-formlike architectural requirements of crRNAs reflect on the unique co-evolution of bacterial CRISPR-Cas systems to use RNA as guides $^{14,105,106}$. Complete chemical modification of guide RNAs, such as for therapeutic CRISPR-based editing, will require addressing the $2^{\prime}$-OH bottleneck that has finally become apparent for Cas9 and Cas12a, and probably for other Cas enzymes. Nonetheless, our results combined with previous studies now provide a roadmap to near-complete chemical modification across the entire Cas12a crRNA. We propose that a primarily $2^{\prime}-\mathrm{F}$ and 2'-O-methyl oligonucleotide, with some DNA or $2^{\prime}-\mathrm{NH}_{2}$ nucleotides, combined with selective PS modification, may be sufficient to provide drug-like profiles with favorable editing activity. Exploring a greater diversity of modifications at wellplaced positions may further unlock efficient editing with little or no native RNA residues. In addition, other properties of Cas12a will need to be investigated, such as the impact of chemical modification schemes on off-target editing, as heavier modification is incorporated.

A recurring theme in crRNA chemical modification studies, especially with Cas9, is that heavy modification schemes successful for in vitro cleavage often do not translate into high editing efficiency in cells ${ }^{61,70,71,80}$. For example, all RNA nucleotides can be replaced with $2^{\prime}-\mathrm{F}$ in the Cas $9 \operatorname{crRNA}^{71}$, and now the Cas12a pseudoknot, and provide very high cleavage in vitro. However, these crRNAs provide little or no editing in cells. Converting predicted 2'-OH polar contact positions back to RNA usually rescues gene editing ${ }^{70,71}$. Thus, it appears that intrinsic biochemical activity is not dependent on $2^{\prime}-\mathrm{OH}$ contacts in bulk reactions in vitro but these become critical within cells, perhaps due to low biochemical RNP concentrations, more complex chromosomal DNA targets, or other unexplored biochemical or cellular factors ${ }^{107}$. Experiments like precision biochemical, thermodynamic, and structural studies, as well as sophisticated single-molecule or gene editing assays in cells, may be necessary to fully understand this phenomenon and unlock "RNA-free" CRISPR-Cas editing for advanced therapeutic control.

The discovery of non-sequence-specific ssDNase cleavage, or trans activity, by Cas12a was quickly converted into an amplification-based diagnostic method ${ }^{41}$. After sequence-specific binding and cutting (cis cleavage) of a DNA target, the catalytic domain of Cas12a is proposed to become solvent exposed such that ssDNA is accessible to the active site ${ }^{45,46}$. This results in high turnover degradation of ssDNA. Once DNA target paired to the guide RNA is released, ssDNase cleavage activity should cease as Cas12a returns to a pre-catalytic structural state ${ }^{46}$. Thus, trans cleavage activity of Cas12a appears to be intrinsically tied to its cis cleavage activity, turnover of cis cleavage products, and conformational-state transitions.

In this study, we initially found what appeared to be differences in cis versus trans cleavage activity for a few crRNAs possessing chemically modified pseudoknots. Certain modification patterns or schemes contributed to this effect. Ultimately, the differential cis-trans activity observed here appears to be a consequence of variable cis cleavage compared to catalytic trans turnover rates for certain guides. This can result in variable ratios of cis to trans activity at time points within a certain activity regime. However, trans activity by modified crRNAs still required cleavage of the target antisense DNA strand in a dsDNA target. Thus, the overall mechanism of trans activity was unaffected. The pseudoknot is proposed to influence catalysis by modulation of $A s$ Cas 12 a conformational transitions 45,46 , which may explain how various activity profiles were achieved by modified pseudoknots. If differential cis-trans cleavage activity could be controlled predictably, it might prove useful for some applications. For example, binding to a specific on-target DNA without cleaving (cis activity) but still allowing trans activity, could provide trans activity for long durations and possibly improve diagnostics. Conversely, cis cleavage without activation of trans cleavage might improve the specificity or safety of other applications, like gene editing ${ }^{108}$. Recently, it was reported that extending the $5^{\prime}$ or $3^{\prime}$ ends of the LbCas12a crRNA could increase on-target activity, and especially enhance trans cleavage rates ${ }^{77}$. Thus, multiple types of crRNA modification may be useful for modulating cis specificity and trans turnover.

\section{Methods}

RNA synthesis. DNA oligonucleotides, crRNAs, and DNA-RNA chimeric 5'handle RNAs were synthesized and purified by Integrated DNA Technologies (IDT). Full-length crRNAs, including DNA-RNA and chemically modified crRNA, were ligated using splint ligation (Supplementary Fig. 1 and Supplementary Tables 1 and 2). Chemically modified $5^{\prime}$-handles were custom synthesized and mass confirmed by mass spectrometry (Supplementary Table 2). Custom, chemically synthesized oligonucleotides may be available upon request and after appropriate inter-institutional material transfer agreements are approved. Singlestranded fluorophore-quencher (FQ) DNA substrate was synthesized commercially by IDT.

Oligonucleotide chemical syntheses were carried out using either an ABI 3400 DNA synthesizer (Applied Biosystems) or a MerMade 12 Oligonucleotide synthesizer (BioAutomation) on a Unylinker CPG (ChemGenes) solid support at a $1 \mu \mathrm{mol}$ scale. Conventional 2'-tert-butyl-dimethylsilyl (TBDMS) ribonucleoside, $2^{\prime}$ - 
fluoro-ribonucleoside (2'-FRNA), and 2'-trifluoroacetyl amino phosphoramidites (ChemGenes) were used, along with newly synthesized oxepane phosphoramidites. Phosphoramidites were dissolved in $\mathrm{MeCN}(0.15 \mathrm{M})$ and activated with 5ethylthio-1H-tetrazole $(0.25 \mathrm{M}$ in MeCN). Capping of failed couplings was carried out by the simultaneous delivery of acetic anhydride in pyridine/THF and $\mathrm{N}$-methylimidazole (16\% in THF) and contacting the solid support for $6 \mathrm{~s}$. Oxidation of the phosphite triester intermediates was affected with $0.1 \mathrm{M}$ iodine in pyridine/ $\mathrm{H}_{2} \mathrm{O} / \mathrm{THF}(20 \mathrm{~s})$. A solution of $3 \%$ trichloroacetic acid in THF, delivered over $95 \mathrm{~s}$, was used to deprotect DMTr groups.

Deprotection and cleavage of oligonucleotides from the solid support was achieved by treatment with $1 \mathrm{~mL}$ of cold $29 \%$ aqueous ammonia/ethanol $(3: 1, \mathrm{v} / \mathrm{v})$ for $16 \mathrm{~h}$ at $65^{\circ} \mathrm{C}$ (or $48 \mathrm{~h}$ at room temperature for oxepane-containing oligonucleotides). The samples were centrifuged and the supernatant was transferred to a clean $1.5 \mathrm{~mL}$ microcentrifuge tube and vented for $30 \mathrm{~min}$, chilled on dry ice, and evaporated to dryness. Removal of the $2^{\prime}$-TBDMS protecting groups for the RNA-containing oligonucleotides was achieved by treatment with a $300 \mu \mathrm{L}$ solution of NMP/Et ${ }_{3} \mathrm{~N} / \mathrm{TREAT}-\mathrm{HF}(3: 4: 6, \mathrm{v} / \mathrm{v})$ for $90 \mathrm{~min}$ at $65^{\circ} \mathrm{C}$ (samples in the SJ series were desilylated by exposure to neat TREAT-HF for $48 \mathrm{~h}$ ), followed by quenching with $3 \mathrm{M} \mathrm{NaOAc}$ buffer $(50 \mu \mathrm{L} ; \mathrm{pH} 5.5)$ and precipitation of the crude oligonucleotide from cold butanol $\left(1 \mathrm{~mL},-20^{\circ} \mathrm{C}\right)$. Samples were chilled on dry ice for $30 \mathrm{~min}$ and then centrifuged. After removing the supernatant, the remaining pellet was evaporated to dryness, taken up in autoclaved Milli- $\mathrm{Q}$ water $(1 \mathrm{~mL})$, and filtered.

Crude oligonucleotides were purified by ion exchange (IE) HPLC. cpEGIP-LA oligonucleotides were purified on a Waters 1525 instrument using a Protein-Pak DEAE 5PW anion exchange column $(21.5 \mathrm{~mm} \times 150 \mathrm{~mm})$. A buffer system consisting of buffer A (10 mM NaOAc, $20 \% \mathrm{MeCN}$ in Milli-Q water) and buffer B $(0.5 \mathrm{M}$ $\mathrm{LiClO}_{4}, 10 \mathrm{mM} \mathrm{NaOAc}, 20 \% \mathrm{MeCN}$ in Milli-Q water) was used for analysis and purification. Using a gradient of $0-50 \% \mathrm{LiClO}_{4}$ (buffer B) over $50 \mathrm{~min}(10 \mathrm{~mL} / \mathrm{min}$, $60^{\circ} \mathrm{C}$ ), the desired compounds eluted at around $30 \mathrm{~min}$. cpEGIP-SJ oligonucleotides were purified on an Agilent 1200 Series Instrument using a Protein-Pak DEAE 5PW column $(7.5 \times 75 \mathrm{~mm})$ at a flow rate of $1 \mathrm{~mL} / \mathrm{min}$, with a buffer system consisting of buffer A (Milli-Q water) and buffer B ( $1 \mathrm{M} \mathrm{LiClO}_{4}$ in Milli-Q water) using a method of $0-60 \%$ buffer $\mathrm{B}$ over $37 \mathrm{~min}$ at $60^{\circ} \mathrm{C}$. Following collection of the desired peaks, excess $\mathrm{LiClO}_{4}$ salts were removed using Gel Pak 2.5 size exclusion columns (Glen Research). Purified oligonucleotides were characterized by electrospray ionizationmass spectrometry (Supplementary Table 2) and quantitated by UV spectroscopy. Extinction coefficients were determined using the IDT OligoAnalyzer tool (https:// www.idtdna.com/calc/analyzer). Extinction coefficients for RNA were used for oligonucleotides containing oxepane modifications.

crRNA splint ligation. crRNA guide sequence ( $200 \mathrm{pmol})$, synthesized with a $5^{\prime}$ phosphate, was ligated to $200 \mathrm{pmol}$ of $5^{\prime}$-handle RNA (synthesized with a $3^{\prime}-\mathrm{OH}$ ) using T4 DNA ligase ${ }^{87}$ (Supplementary Table 1) For DNA ligase, 220 pmol splint DNA, complementary to both guide and 5'-handle RNA, was annealed to $200 \mathrm{pmol}$ of each crRNA portion in RNA resuspension buffer ( $5 \mathrm{mM}$ Tris, pH 7.4, $0.5 \mathrm{mM}$ EDTA) at $65^{\circ} \mathrm{C}$, then slow-cooled to room temperature. Ligation was performed using $1 \mu \mathrm{L}$ of concentrated DNA ligase (30 U/ $\mu \mathrm{L})$ (Thermo Scientific, EL0013), $1 \mathrm{x}$ ligation buffer ( $400 \mathrm{mM}$ Tris-HCL, $100 \mathrm{mM} \mathrm{MgCl} 2,100 \mathrm{mM}$ DTT, $5 \mathrm{mM}$ ATP), and $0.5 \mu \mathrm{L}$ of SUPERase-In (Invitrogen) in a final volume of $100 \mu \mathrm{L}$. The reaction was incubated for $90 \mathrm{~min}$ at $37^{\circ} \mathrm{C}$ and stopped by adding $1 \mu \mathrm{L}$ of $0.5 \mathrm{M}$ EDTA pH 8 and phenol-chloroform extracted. Ligation products were visualized by resolving on $15 \%$ denaturing polyacrylamide gels and staining with methylene blue. Fulllength ligation products were gel-purified by crush-and-soak elution, phenolchloroform extracted, ethanol precipitated, and quantified by measuring absorbance at $260 \mathrm{~nm}$ and calculated extinction coefficients using nearest neighbor approximations with Beer's Law. Electrospray ionization-mass spectrometry was used to confirm the mass of a few full-length, ligated crRNAs to ensure ligation and gel-purification was proceeding properly.

\section{AsCas12a enzyme expression and purification. Plasmid encoding of an} AsCas12a was obtained from Addgene (79007). AsCas12a proteins were prepared similarly to that previously described for $S p$ Cas $9^{109}$. Briefly, protein expression was induced in Rosetta (DE3) cells grown in Luria-Bertani (LB) broth with $0.2 \mathrm{mM}$ isopropyl thiogalactopyranoside (IPTG) at $18^{\circ} \mathrm{C}$ for $16 \mathrm{~h}$. Cell pellets were resuspended in $6 \mathrm{~mL}$ chilled binding buffer $(20 \mathrm{mM}$ Tris- $\mathrm{HCl}, \mathrm{pH} 8.0,250 \mathrm{mM} \mathrm{NaCl}$, $1 \mathrm{mM}$ PMSF, $5 \mathrm{mM}$ imidazole) per $0.5 \mathrm{~L}$ of culture. Resuspended cells were sonicated and clarified by centrifugation. For the $0.5 \mathrm{~L}$ of culture, $5 \mathrm{~mL}$ His-Pur CobaltCMA resin (Thermo Scientific) was equilibrated with binding buffer and the supernatant added to the equilibrated resin and incubated at $4{ }^{\circ} \mathrm{C}$ for $1 \mathrm{~h}$ with inversion to mix every $15 \mathrm{~min}$. The column was washed sequentially with at least 10 bed volumes of increasing concentrations of $\mathrm{NaCl}$ in wash buffer $(20 \mathrm{mM}$ Tris$\mathrm{HCl}, \mathrm{pH} 8.0,10 \mathrm{mM}$ imidazole, $0.25 / 0.5 / 0.75 / 1.0 \mathrm{M} \mathrm{NaCl}$ ). Protein was eluted with $130 \mathrm{mM}$ imidazole buffer $(20 \mathrm{mM}$ Tris- $\mathrm{HCl}, \mathrm{pH} 8.0,250 \mathrm{mM} \mathrm{NaCl}, 200 \mathrm{mM}$ imidazole). Purified AsCas12a enzyme was concentrated and buffer exchanged using centrifugal concentrators (Sartorius, 30,000 MWCO) into 2x storage buffer ( $40 \mathrm{mM}$ Tris, $\mathrm{pH} 7.5,300 \mathrm{mM} \mathrm{KCl}, 1 \mathrm{mM}$ EDTA, and $2 \mathrm{mM} \mathrm{DTT}$ ). Then one volume of glycerol added to obtain a final of $50 \%$ glycerol. Protein stocks were then stored at $-80^{\circ} \mathrm{C}$. Concentration of $A s$ Cas $12 \mathrm{a}$ was determined by UV absorbance at $280 \mathrm{~nm}$ using a calculated extinction coefficient and Beer's law.
In vitro AsCas12a Cis cleavage activity assays. A $1 \mathrm{~kb}$ fragment of the target EGFP gene was PCR-amplified using "cpEGIP_vitro targ" primers (Supplementary Table 1) from plasmid DNA (Addgene, 26777) and purified by phenol-chloroform extraction and ethanol precipitation. Target DNA $(185 \mathrm{ng})$ was spotted into tubes and combined with the AsCas12a $(0.75 \mu \mathrm{M}$ final $)$ and crRNA $(0.3 \mu \mathrm{M}$ final $)$ in $1 \mathrm{x}$ cleavage buffer ( $20 \mathrm{mM}$ Tris- $\mathrm{HCl}, \mathrm{pH} 7.5,100 \mathrm{mM} \mathrm{KCl,} 5 \%$ glycerol, $1 \mathrm{mM}$ DTT, $0.5 \mathrm{mM}$ EDTA, $2 \mathrm{mM} \mathrm{MgCl}_{2}$ ) supplemented with $0.1 \mathrm{mg} / \mathrm{mL}$ of purified yeast tRNA. Molar excess of the AsCas12a over crRNA allows RNP concentration approximation by using crRNA concentration. Although a fraction of the Cas enzyme remains unbound, we have previously found this approach to be an accurate method for predicting the concentration of actual assembled RNP complexes ${ }^{69,71}$. The $40 \mu \mathrm{L}$ reaction was incubated at $37^{\circ} \mathrm{C}$ for $2 \mathrm{~h}$. The reaction was then treated with $10 \mu \mathrm{g}$ of RNase A (Thermo Scientific) for $15 \mathrm{~min}$ followed by $20 \mu \mathrm{g}$ of Proteinase K (Thermo Scientific) for $15 \mathrm{~min}$ at room temperature. The reaction products were then precipitated in 10 volumes of $2 \% \mathrm{LiClO}_{4}$ in acetone for $>1 \mathrm{~h}$ at $-20^{\circ} \mathrm{C}$. Precipitated reactions were centrifuged and washed with acetone, air dried, and resuspended in gel-loading dye ( $10 \%$ glycerol, $1 \mathrm{x}$ TBE, orange $\mathrm{G}$ dye) and resolved on $1.5 \%$ agarose gels. Agarose gels were stained with ethidium bromide and visualized using a UV imager. The fractions of target cleaved versus uncleaved were quantified using ImageJ software (v1.43u). Dot plot data points represent experimental replicates, not technical replicates.

For cleavage time-course assays, AsCas12a and crRNA (cpEGIP or cpEGIPSJ14) were assembled at room temperature for $10 \mathrm{~min}$. The RNP was then added to a target DNA in at $37^{\circ} \mathrm{C}$ in a final of $1 \mathrm{x}$ cleavage buffer and reactions stopped at indicated time points by precipitation with $2 \% \mathrm{LiClO}_{4}$ in acetone and incubated at $-20^{\circ} \mathrm{C}$. Samples were pelleted by centrifugation then washed with acetone. After samples were air dried, they were resuspended in $1 \mathrm{x}$ loading dye ( $10 \%$ glycerol, $1 \mathrm{x}$ TBE, orange $\mathrm{G}$ dye), treated with RNase $\mathrm{A}$ and proteinase $\mathrm{K}$ as described above, and cleavage products resolved on $1.5 \%$ agarose gels. Quantification was performed as described above.

\section{Generation of HEK293T cells stably expressing EGFP and AsCas12a.}

HEK293T cells stably expressing EGFP were a kind gift from Dr. Wen Xue ${ }^{61}$. We subcloned AsCas12a from pY010 (pcDNA3.1-hAsCpf1) (Addgene, 69982) into pLJM1-EGFP lentivector ${ }^{110}$ (Addgene, 19319) such that the EGFP gene was replaced with AsCas12a. Subcloning was performed with NheI and EcoRI restriction enzymes and resulted in retention of the C-terminal NLS and HA tag on $A s C p f 1$ in the new vector, pLJM1-AsCas12a (Supplementary Fig. 2a). The PGKdriven puromycin resistance gene was replaced with a hygromycin resistance gene downstream of an internal ribosomal entry site. HEK293T cells were transfected with pLJM1-AsCas12a, pCMV-VSVG (Addgene, 8454), and pCMV-dR8.2 dvpr (Addgene, 8455) ${ }^{111}$ using the calcium phosphate technique. Medium was replaced $18 \mathrm{~h}$ after transfection. Lentiviral containing medium was collected $48 \mathrm{~h}$ later and centrifuged at $1000 \mathrm{~g}$ for $5 \mathrm{~min}$. Hexadimethrine bromide was added to the medium $(8 \mu \mathrm{g} / \mathrm{mL})$ and the medium was used to infect HEK293T-EGFP cells on 6-well plates for $8 \mathrm{~h}$.

Transfection of cells with pLJM1-AsCas12a and staining with anti-HA (Santa Cruz Biotechnology, 1:1000) revealed primarily cytoplasmic localization (Supplementary Fig. 2b). Therefore, we cloned an additional 3x NLS tag onto the $\mathrm{N}$-terminus of AsCas12a to generate pLJM1-3xNLS-AsCas12a. Staining with antiHA revealed exclusively nuclear staining (Supplementary Fig. 2b). Stably integrated cells were selected using $100 \mu \mathrm{g} / \mathrm{mL}$ hygromycin and a clonal cell line was expanded. Expression and nuclear localization of $A s$ Cas12a was confirmed in the stable cell line (Supplementary Fig. 3c). Stable pLJM1-3xNLS-AsCas12a HEK293TEGFP cells may be available upon request and after appropriate inter-institutional material transfer agreements are approved.

\section{Cell-based editing measured by flow cytometry. HEK293T cells expressing} EGFP and $A s$ Cas12a were grown in Dulbecco's modified eagle's medium (DMEM) with $1 \mathrm{x}$ non-essential amino acids (NEAA), $5 \%$ cosmic calf serum (CCS) and $2.5 \%$ fetal bovine serum (FBS) without antibiotics. Cells were reverse-transfected $(40,000$ cells) in six experimental replicates in 96-well plates with 20 pmol of crRNA and $0.3 \mu \mathrm{L}$ RNAiMAX lipid (Invitrogen) in a final reaction of $200 \mu \mathrm{L}$ of OptiMEM. After $8 \mathrm{~h}$, one volume of media containing 5\% FBS and 5\% CCS was added to cells and further incubated overnight. Media was then replaced with full media and cells grown for an additional 4 days. For flow cytometry, cells were washed with $200 \mu \mathrm{L}$ phosphate-buffered saline (PBS) and trypsinized by adding $70 \mu \mathrm{L}$ of trypsin-EDTA solution. Then, $100 \mu \mathrm{L}$ of media was added to the cells. The cells were spun for $5 \mathrm{~min}$ at $300 \mathrm{~g}$ at room temperature. Cells were washed again with $200 \mu \mathrm{L}$ PBS, resuspended in $200 \mu \mathrm{L}$ PBS and counted in an Attune flow cytometer. EGFP was detected using the blue laser (BL1 channel). At least 20,000 events were collected and analyzed by Attune software (v3.12). The cells were gated based on forward and side scattering (FSC-A/SSC-A) to remove cell debris, gated to select single cells, and gated to select EGFP-positive cells. The quadrant gate was established using the signal from non-EGFP-expressing control cells. Untreated HEK293T cells expressing EGFP and AsCas12a contained $\sim 5 \%$ non-fluorescent cells (Fig. S7). The average from five or six replicates (see figure legends) was used for background subtraction to determine the extent of cell-based editing after treatment. Two crRNAs with different guide sequences, cpEGIP and cpEGIPe, were initially screened. cpEGIPe provided substantial editing activity $(75-80 \%)$ and was chosen 
for subsequent modification and editing experiments in cells (Supplementary Fig. 2d).

Fluorophore-quencher (FQ) Trans cleavage reporter assay. AsCas12a and crRNA were preassembled into RNP complex by incubating $500 \mathrm{nM}$ AsCas12a with $550 \mathrm{nM}$ crRNA and $25 \mathrm{nM}$ DNA target in $1 \mathrm{X}$ cleavage buffer $(20 \mathrm{mM}$ Tris$\mathrm{HCl}, \mathrm{pH}$ 7.5, $100 \mathrm{mM} \mathrm{KCl}, 5 \%$ glycerol, $1 \mathrm{mM}$ DTT, $0.5 \mathrm{mM}$ EDTA, $2 \mathrm{mM} \mathrm{MgCl}_{2}$ ) on ice for $15 \mathrm{~min}$. Certain experiments either omitted target DNA, crRNA, or used PS-modified target at the same concentrations as indicated above. Reactions were initiated by adding FQ ssDNA substrate to a final of $3 \mu \mathrm{M}$ and placing the plate in a Bio-Rad CFX96 instrument with the block set at $4{ }^{\circ} \mathrm{C}$. The block was cycled to $37^{\circ} \mathrm{C}$ and the plate was read using the SYBR-only channel every $53 \mathrm{~s}$ (reading takes $7 \mathrm{~s}$ ) such that fluorescence readings were collected at $1 \mathrm{~min}$ intervals. Fluorescence was collected for up to $150 \mathrm{~min}$. Maximum fluorescence values at $1 \mathrm{~h}$ were used to compare non-sequence-specific trans cleavage activity of varying crRNAs containing modified $5^{\prime}$ pseudoknots. Error was calculated by standard deviation, representing three or more experimental replicates.

For strand-nicking cleavage assays utilizing radiolabeled target DNA strands, individual DNA strands (cpEGIPe target sequence) were $5^{\prime}$ radiolabeled with $\mathrm{T} 4$ polynucleotide kinase and ${ }^{32} \mathrm{P}-\gamma$-ATP as previously described ${ }^{112}$. Radiolabeled DNA strands were then gel-purified from a 15\% denaturing Urea-PAGE by crushand-soak elution after visualizing radioactive bands by autoradiography. Duplexes with differentially radiolabeled sense (non-target) or antisense (target) strands were annealed and slow-cooled with a $\sim 1.2$-fold molar excess of unlabeled complementary strand. Reaction conditions then utilized those of a standard trans cleavage assay but lacking a FQ-labeled ssDNA substrate. Reactions proceeded for $1 \mathrm{~h}$ before being stopped by phenol-chloroform extraction. Cleavage products were resolved on a $15 \%$ denaturing PAGE and exposed to phosphorimager for visualization.

Limited trypsin hydrolysis of AsCas12a protein and RNP. Trypsin proteolysis was performed using $30 \mu \mathrm{g}$ of AsCas12a in the presence and absence of crRNA and double-stranded target DNA (1:1.5:2 molar ratio $)^{113}$. The RNP was incubated at room temperature for $5 \mathrm{~min}$. The resulting samples were incubated with TrypsinEDTA solution $(0.05 \%$, Invitrogen) at a mass ratio of $100: 1$ and the partial proteolysis was conducted at $37^{\circ} \mathrm{C}$ for $15 \mathrm{~min}$. The reaction was stopped by the addition of SDS-PAGE loading buffer and heating the samples for $5 \mathrm{~min}$ at $95^{\circ} \mathrm{C}$. The reaction products were analyzed by $12 \%$ SDS-PAGE and stained with Coomassie brilliant blue G-250 in 50\% (v/v) methanol and 10\% (v/v) acetic acid then destained in the same solution without dye.

Serum stability of modified Cas12a pseudoknots and crRNAs. Serum stability test was performed using 300 pmol of pseudoknot or crRNA and $5 \mu \mathrm{L}$ of $10 \%$ FBS in $1 \mathrm{x}$ PBS in a total reaction volume of $50 \mu \mathrm{L}$. Reactions were incubated at $37^{\circ} \mathrm{C}$ and then stopped after $2 \mathrm{~h}$ by adding $1 \mu \mathrm{L}$ of $0.5 \mathrm{M}$ EDTA. RNA was extracted by phenol-chloroform extraction, precipitated with $2 \% \mathrm{LiClO}_{4}$, and resolved on a $15 \%$ TBE-buffered denaturing (7 M urea) polyacrylamide gel. Gels were stained with methylene blue to visualize digestion patterns.

Reporting summary. Further information on research design is available in the Nature Research Reporting Summary linked to this article.

\section{Data availability}

Any additional data or materials can be obtained upon reasonable request. Source data are provided with this paper.

Received: 27 October 2020; Accepted: 1 November 2021; Published online: 15 November 2021

\section{References}

1. Bolotin, A., Quinquis, B., Sorokin, A. \& Ehrlich, S. D. Clustered regularly interspaced short palindrome repeats (CRISPRs) have spacers of extrachromosomal origin. Microbiology 151, 2551-2561 (2005).

2. Mojica, F. J., Diez-Villasenor, C., Garcia-Martinez, J. \& Soria, E. Intervening sequences of regularly spaced prokaryotic repeats derive from foreign genetic elements. J. Mol. Evol. 60, 174-182 (2005).

3. Barrangou, R. et al. CRISPR provides acquired resistance against viruses in prokaryotes. Science 315, 1709-1712 (2007).

4. Brouns, S. J. et al. Small CRISPR RNAs guide antiviral defense in prokaryotes. Science 321, 960-964 (2008).

5. Tyson, G. W. \& Banfield, J. F. Rapidly evolving CRISPRs implicated in acquired resistance of microorganisms to viruses. Environ. Microbiol. 10, 200-207 (2008).
6. Garneau, J. E. et al. The CRISPR/Cas bacterial immune system cleaves bacteriophage and plasmid DNA. Nature 468, 67-71 (2010).

7. Gasiunas, G., Barrangou, R., Horvath, P. \& Siksnys, V. Cas9-crRNA ribonucleoprotein complex mediates specific DNA cleavage for adaptive immunity in bacteria. Proc. Natl Acad. Sci. USA 109, E2579-E2586 (2012).

8. Jinek, M. et al. A programmable dual-RNA-guided DNA endonuclease in adaptive bacterial immunity. Science 337, 816-821 (2012).

9. Wiedenheft, B., Sternberg, S. H. \& Doudna, J. A. RNA-guided genetic silencing systems in bacteria and archaea. Nature 482, 331-338 (2012).

10. Anders, C., Niewoehner, O., Duerst, A. \& Jinek, M. Structural basis of PAMdependent target DNA recognition by the Cas9 endonuclease. Nature 513, 569-573 (2014)

11. Jinek, M. et al. Structures of Cas 9 endonucleases reveal RNA-mediated conformational activation. Science 343, 1247997 (2014).

12. Nishimasu, H. et al. Crystal structure of Cas9 in complex with guide RNA and target DNA. Cell 156, 935-949 (2014).

13. Nunez, J. K. et al. Cas1-Cas 2 complex formation mediates spacer acquisition during CRISPR-Cas adaptive immunity. Nat. Struct. Mol. Biol. 21, 528-534 (2014).

14. Makarova, K. S. et al. An updated evolutionary classification of CRISPR-Cas systems. Nat. Rev. Microbiol. 13, 722-736 (2015).

15. Nishimasu, H. et al. Crystal structure of Staphylococcus aureus Cas9. Cell 162, 1113-1126 (2015)

16. Zetsche, B. et al. Cpf1 is a single RNA-guided endonuclease of a class 2 CRISPR-Cas system. Cell 163, 759-771 (2015)

17. Abudayyeh, O. O. et al. C2c2 is a single-component programmable RNAguided RNA-targeting CRISPR effector. Science 353, aaf5573 (2016).

18. Kim, E. et al. In vivo genome editing with a small Cas 9 orthologue derived from Campylobacter jejuni. Nat. Commun. 8, 14500 (2017).

19. Shah, S. A., Erdmann, S., Mojica, F. J. \& Garrett, R. A. Protospacer recognition motifs: mixed identities and functional diversity. RNA Biol. 10, 891-899 (2013).

20. Yamano, T. et al. Crystal structure of Cpfl in complex with guide RNA and target DNA. Cell 165, 949-962 (2016).

21. Gao, L. et al. Engineered Cpf1 variants with altered PAM specificities. Nat. Biotechnol. 35, 789-792 (2017).

22. Cong, L. et al. Multiplex genome engineering using CRISPR/Cas systems. Science 339, 819-823 (2013).

23. Mali, P. et al. RNA-guided human genome engineering via Cas9. Science 339, 823-826 (2013)

24. Qi, L. S. et al. Repurposing CRISPR as an RNA-guided platform for sequencespecific control of gene expression. Cell 152, 1173-1183 (2013).

25. Doudna, J. A. \& Charpentier, E. Genome editing. The new frontier of genome engineering with CRISPR-Cas9. Science 346, 1258096 (2014).

26. Dai, W. J. et al. CRISPR-Cas9 for in vivo gene therapy: promise and hurdles. Mol. Ther. Nucleic Acids 5, e349 (2016).

27. Monteys, A. M., Ebanks, S. A., Keiser, M. S. \& Davidson, B. L. CRISPR/Cas editing of the mutant Huntingtin allele in vitro and in vivo. Mol. Ther. 25, 12-23 (2017).

28. Pineda, M., Moghadam, F., Ebrahimkhani, M. R. \& Kiani, S. Engineered CRISPR systems for next generation gene therapies. ACS Synth. Biol. 6, 1614-1626 (2017)

29. Conboy, I., Murthy, N., Etienne, J. \& Robinson, Z. Making gene editing a therapeutic reality. F1000Research 7, 1970 (2018).

30. Vojta, A. et al. Repurposing the CRISPR-Cas9 system for targeted DNA methylation. Nucleic Acids Res. 44, 5615-5628 (2016).

31. Komor, A. C., Kim, Y. B., Packer, M. S., Zuris, J. A. \& Liu, D. R. Programmable editing of a target base in genomic DNA without doublestranded DNA cleavage. Nature 533, 420-424 (2016).

32. Hur, J. K. et al. Targeted mutagenesis in mice by electroporation of Cpf1 ribonucleoproteins. Nat. Biotechnol. 34, 807-808 (2016).

33. Zetsche, B. et al. Multiplex gene editing by CRISPR-Cpfl using a single crRNA array. Nat. Biotechnol. 35, 31-34 (2017).

34. Kim, D. et al. Genome-wide analysis reveals specificities of Cpf1 endonucleases in human cells. Nat. Biotechnol. 34, 863-868 (2016).

35. Kleinstiver, B. P. et al. Genome-wide specificities of CRISPR-Cas Cpf1 nucleases in human cells. Nat. Biotechnol. 34, 869-874 (2016).

36. Kim, S., Kim, D., Cho, S. W., Kim, J. \& Kim, J. S. Highly efficient RNA-guided genome editing in human cells via delivery of purified Cas9 ribonucleoproteins. Genome Res. 24, 1012-1019 (2014).

37. O'Geen, H., Henry, I. M., Bhakta, M. S., Meckler, J. F. \& Segal, D. J. A genomewide analysis of Cas9 binding specificity using ChIP-seq and targeted sequence capture. Nucleic Acids Res. 43, 3389-3404 (2015).

38. Wang, S. et al. No off-target mutations in functional genome regions of a CRISPR/Cas9-generated monkey model of muscular dystrophy. J. Biol. Chem. 293, 11654-11658 (2018).

39. Dong, D. et al. The crystal structure of Cpf1 in complex with CRISPR RNA Nature 532, 522-526 (2016). 
40. Leenay, R. T. et al. Identifying and visualizing functional PAM diversity across CRISPR-Cas systems. Mol. Cell 62, 137-147 (2016).

41. Chen, J. S. et al. CRISPR-Cas12a target binding unleashes indiscriminate single-stranded DNase activity. Science 360, 436-439 (2018).

42. Li, S. Y. et al. CRISPR-Cas12a has both cis- and trans-cleavage activities on single-stranded DNA. Cell Res. 28, 491-493 (2018).

43. Nouri, R., Jiang, Y., Lian, X. L. \& Guan, W. Sequence-specific recognition of HIV-1 DNA with solid-state CRISPR-Cas12a-assisted nanopores (SCAN). ACS Sens. 5, 1273-1280 (2020).

44. Broughton, J. P. et al. CRISPR-Cas12-based detection of SARS-CoV-2. Nat. Biotechnol. 38, 870-874 (2020).

45. Swarts, D. C. \& Jinek, M. Mechanistic insights into the cis- and trans-acting DNase activities of Cas12a. Mol. Cell 73, 589-600.e584 (2019).

46. Stella, S. et al. Conformational activation promotes CRISPR-Cas12a catalysis and resetting of the endonuclease activity. Cell 175, 1856-1871.e1821 (2018).

47. Gagnon, K. T. \& Corey, D. R. Stepping toward therapeutic CRISPR. Proc. Natl Acad. Sci. USA 112, 15536-15537 (2015).

48. Beaudet, A. L. \& Meng, L. Gene-targeting pharmaceuticals for single-gene disorders. Hum. Mol. Genet. 25, R18-R26 (2016).

49. Shin, J. W. et al. Permanent inactivation of Huntington's disease mutation by personalized allele-specific CRISPR/Cas9. Hum. Mol. Genet. 25, 4566-4576 (2016).

50. Fellmann, C., Gowen, B. G., Lin, P. C., Doudna, J. A. \& Corn, J. E. Cornerstones of CRISPR-Cas in drug discovery and therapy. Nat. Rev. Drug Discov. 16, 89-100 (2017).

51. Hynes, R. O., Coller, B. S. \& Porteus, M. Toward responsible human genome editing. JAMA 317, 1829-1830 (2017).

52. Shim, G. et al. Therapeutic gene editing: delivery and regulatory perspectives. Acta Pharmacologica Sin. 38, 738-753 (2017).

53. Song, M. The CRISPR/Cas9 system: their delivery, in vivo and ex vivo applications and clinical development by startups. Biotechnol. Prog. 33, 1035-1045 (2017).

54. Wilson, R. C. \& Gilbert, L. A. The promise and challenge of in vivo delivery for genome therapeutics. ACS Chem. Biol. 13, 376-382 (2017).

55. Karimian, A. et al. CRISPR/Cas9 technology as a potent molecular tool for gene therapy. J. Cell. Physiol. 234, 12267-12277 (2019).

56. Skipper, K. A. \& Mikkelsen, J. G. Toward in vivo gene therapy using CRISPR. Methods Mol. Biol. 1961, 293-306 (2019).

57. Chew, W. L. Immunity to CRISPR Cas9 and Cas12a therapeutics. Wiley Interdiscip. Rev. Syst. Biol. Med. 10, e1408 (2018).

58. Dowdy, S. F. Overcoming cellular barriers for RNA therapeutics. Nat Biotechnol. 35, 222-229 (2017).

59. O'Geen, H., Yu, A. S. \& Segal, D. J. How specific is CRISPR/Cas9 really? Curr. Opin. Chem. Biol. 29, 72-78 (2015).

60. Wang, M. et al. Efficient delivery of genome-editing proteins using bioreducible lipid nanoparticles. Proc. Natl Acad. Sci. USA 113, 2868-2873 (2016).

61. Yin, H. et al. Structure-guided chemical modification of guide RNA enables potent non-viral in vivo genome editing. Nat. Biotechnol. 35, 1179-1187 (2017).

62. Miller, J. B. et al. Non-viral CRISPR/Cas gene editing in vitro and in vivo enabled by synthetic nanoparticle co-delivery of Cas9 mRNA and sgRNA. Angew. Chem. Int. Ed. Engl. 56, 1059-1063 (2017).

63. Cheng, Q. et al. Selective organ targeting (SORT) nanoparticles for tissuespecific mRNA delivery and CRISPR-Cas gene editing. Nat. Nanotechnol. 15, 313-320 (2020).

64. Kowalski, P. S., Rudra, A., Miao, L. \& Anderson, D. G. Delivering the messenger: advances in technologies for therapeutic mRNA delivery. Mol. Ther. 27, 710-728 (2019)

65. Meade, B. R. \& Dowdy, S. F. The road to therapeutic RNA interference (RNAi): tackling the 800 pound siRNA delivery gorilla. Discov. Med. 8, 253-256 (2009).

66. Khvorova, A. \& Watts, J. K. The chemical evolution of oligonucleotide therapies of clinical utility. Nat. Biotechnol. 35, 238-248 (2017).

67. Smith, C. I. E. \& Zain, R. Therapeutic oligonucleotides: state of the art. Annu. Rev. Pharmacol. Toxicol. 59, 605-630 (2019).

68. Filippova, J., Matveeva, A., Zhuravlev, E. \& Stepanov, G. Guide RNA modification as a way to improve CRISPR/Cas9-based genome-editing systems. Biochimie 167, 49-60 (2019).

69. Kartje, Z. J., Barkau, C. L., Rohilla, K. J., Ageely, E. A. \& Gagnon, K. T. Chimeric guides probe and enhance Cas9 biochemical activity. Biochemistry 57, 3027-3031 (2018).

70. Mir, A. et al. Heavily and fully modified RNAs guide efficient SpyCas9mediated genome editing. Nat. Commun. 9, 2641 (2018)

71. O'Reilly, D. et al. Extensive CRISPR RNA modification reveals chemical compatibility and structure-activity relationships for Cas9 biochemical activity. Nucleic Acids Res. 47, 546-558 (2019).
72. Hendel, A. et al. Chemically modified guide RNAs enhance CRISPR-Cas genome editing in human primary cells. Nat. Biotechnol. 33, 985-989 (2015).

73. Basila, M., Kelley, M. L. \& Smith, A. V. B. Minimal 2'-O-methyl phosphorothioate linkage modification pattern of synthetic guide RNAs for increased stability and efficient CRISPR-Cas9 gene editing avoiding cellular toxicity. PLoS ONE 12, e0188593 (2017).

74. Li, B. et al. Engineering CRISPR-Cpf1 crRNAs and mRNAs to maximize genome editing efficiency. Nat. Biomed. Eng. 1, 0066 (2017).

75. Park, H. M. et al. Extension of the crRNA enhances $\mathrm{Cpf1}$ gene editing in vitro and in vivo. Nat. Commun. 9, 3313 (2018).

76. Ha, D. I. et al. Highly efficient and safe genome editing by CRISPR-Cas12a using CRISPR RNA with a ribosyl-2'-O-methylated uridinylate-rich $3^{\prime}$ overhang in mouse zygotes. Exp. Mol. Med. 52, 1823-1830 (2020).

77. Nguyen, L. T., Smith, B. M. \& Jain, P. K. Enhancement of trans-cleavage activity of Cas12a with engineered crRNA enables amplified nucleic acid detection. Nat. Commun. 11, 4906 (2020).

78. Kim, H. et al. Enhancement of target specificity of CRISPR-Cas12a by using a chimeric DNA-RNA guide. Nucleic Acids Res. 48, 8601-8616 (2020).

79. Yin, H. et al. Partial DNA-guided Cas9 enables genome editing with reduced off-target activity. Nat. Chem. Biol. 14, 311-316 (2018).

80. Rueda, F. O. et al. Mapping the sugar dependency for rational generation of a DNA-RNA hybrid-guided Cas9 endonuclease. Nat. Commun. 8, 1610 (2017).

81. McMahon, M. A., Prakash, T. P., Cleveland, D. W., Bennett, C. F. \& Rahdar, M. Chemically modified Cpf1-CRISPR RNAs mediate efficient genome editing in mammalian cells. Mol. Ther. 26, 1228-1240 (2018).

82. Rahdar, M. et al. Synthetic CRISPR RNA-Cas9-guided genome editing in human cells. Proc. Natl Acad. Sci. USA 112, E7110-E7117 (2015).

83. Ryan, D. E. et al. Improving CRISPR-Cas specificity with chemical modifications in single-guide RNAs. Nucleic Acids Res. 46, 792-803 (2018).

84. Cromwell, C. R. et al. Incorporation of bridged nucleic acids into CRISPR RNAs improves Cas9 endonuclease specificity. Nat. Commun. 9, 1448 (2018).

85. Liu, F. \& Theimer, C. A. Telomerase activity is sensitive to subtle perturbations of the TLC1 pseudoknot $3^{\prime}$ stem and tertiary structure. J. Mol. Biol. 423, 719-735 (2012).

86. Ferre-D'Amare, A. R. \& Doudna, J. A. RNA folds: insights from recent crystal structures. Annu. Rev. Biophys. Biomol. Struct. 28, 57-73 (1999).

87. Kershaw, C. J. \& O'Keefe, R. T. Splint ligation of RNA with T4 DNA ligase. Methods Mol. Biol. 941, 257-269 (2012)

88. Ui-Tei, K. et al. Functional dissection of siRNA sequence by systematic DNA substitution: modified siRNA with a DNA seed arm is a powerful tool for mammalian gene silencing with significantly reduced off-target effect. Nucleic Acids Res. 36, 2136-2151 (2008).

89. Salazar, M., Fedoroff, O. Y., Miller, J. M., Ribeiro, N. S. \& Reid, B. R. The DNA strand in DNA.RNA hybrid duplexes is neither B-form nor A-form in solution. Biochemistry 32, 4207-4215 (1993).

90. Fu, L. et al. DNA pseudoknot-functionalized sensing platform for chemoselective analysis of mercury ions. Analyst 137, 4425-4427 (2012),

91. Baker, E. S., Dupuis, N. F. \& Bowers, M. T. DNA hairpin, pseudoknot, and cruciform stability in a solvent-free environment. J. Phys. Chem. B 113, 1722-1727 (2009)

92. Patra, A. et al. 2'-Fluoro RNA shows increased Watson-Crick H-bonding strength and stacking relative to RNA: evidence from NMR and thermodynamic data. Angew. Chem. Int Ed. Engl. 51, 11863-11866 (2012).

93. Forconi, M. et al. $2^{\prime}$-Fluoro substituents can mimic native $2^{\prime}$-hydroxyls within structured RNA. Chem. Biol. 18, 949-954 (2011).

94. Habibian, M. et al. Seven-membered ring nucleoside analogues: stereoselective synthesis and studies on their conformational properties. Org. Lett. 17, 5416-5419 (2015)

95. Martin-Pintado, N. et al. The solution structure of double helical arabino nucleic acids (ANA and 2'F-ANA): effect of arabinoses in duplex-hairpin interconversion. Nucleic Acids Res. 40, 9329-9339 (2012).

96. Aurup, H., Tuschl, T., Benseler, F., Ludwig, J. \& Eckstein, F. Oligonucleotide duplexes containing $2^{\prime}$-amino- $2^{\prime}$-deoxycytidines: thermal stability and chemical reactivity. Nucleic Acids Res. 22, 20-24 (1994).

97. Williams, D. M., Pieken, W. A. \& Eckstein, F. Function of specific $2^{\prime}$-hydroxyl groups of guanosines in a hammerhead ribozyme probed by $2^{\prime}$ modifications. Proc. Natl Acad. Sci. USA 89, 918-921 (1992).

98. Jellinek, D. et al. Potent $2^{\prime}$-amino-2'-deoxypyrimidine RNA inhibitors of basic fibroblast growth factor. Biochemistry 34, 11363-11372 (1995).

99. Yan, X., Gao, X. \& Zhang, Z. Isolation and characterization of $2^{\prime}$-aminomodified RNA aptamers for human TNFalpha. Genom. Proteom. Bioinf. 2 , $32-42$ (2004).

100. Nilsen, T. W. Nucleotide analog interference mapping. Cold Spring Harb. Protoc. 2015, 604-608 (2015)

101. Westheimer, F. H. Why nature chose phosphates. Science 235, 1173-1178 (1987).

102. Shmakov, S. et al. Diversity and evolution of class 2 CRISPR-Cas systems. Nat Rev. Microbiol. 15, 169-182 (2017). 
103. Swarts, D. C., van der Oost, J. \& Jinek, M. Structural basis for guide RNA processing and seed-dependent DNA targeting by CRISPR-Cas12a. Mol. Cell 66, 221-233.e224 (2017).

104. Deleavey, G. F. \& Damha, M. J. Designing chemically modified oligonucleotides for targeted gene silencing. Chem. Biol. 19, 937-954 (2012).

105. Huttenhofer, A. \& Schattner, P. The principles of guiding by RNA: chimeric RNA-protein enzymes. Nat. Rev. Genet. 7, 475-482 (2006).

106. Chylinski, K., Makarova, K. S., Charpentier, E. \& Koonin, E. V. Classification and evolution of type II CRISPR-Cas systems. Nucleic Acids Res. 42, 6091-6105 (2014).

107. Strohkendl, I. et al. Inhibition of CRISPR-Cas12a DNA targeting by nucleosomes and chromatin. Sci. Adv. 7, eabd6030 (2021).

108. Murugan, K., Seetharam, A. S., Severin, A. J. \& Sashital, D. G. CRISPR-Cas12a has widespread off-target and dsDNA-nicking effects. J. Biol. Chem. 295, 5538-5553 (2020).

109. Anders, C. \& Jinek, M. In vitro enzymology of Cas9. Methods Enzymol. 546, 1-20 (2014).

110. Sancak, Y. et al. The Rag GTPases bind raptor and mediate amino acid signaling to mTORC1. Science 320, 1496-1501 (2008).

111. Stewart, S. A. et al. Lentivirus-delivered stable gene silencing by RNAi in primary cells. RNA 9, 493-501 (2003).

112. Habibian, M. et al. Effect of $2^{\prime}-5^{\prime} / 3^{\prime}-5^{\prime}$ phosphodiester linkage heterogeneity on RNA interference. Nucleic Acids Res. 48, 4643-4657 (2020).

113. Jiang, F., Zhou, K., Ma, L., Gressel, S. \& Doudna, J. A. A Cas9-guide RNA complex preorganized for target DNA recognition. Science 348, 1477-1481 (2015).

\section{Acknowledgements}

This work was supported by startup funding to K.T.G. and a grant from the National Institutes of Health (1 R01 GM135646-01) to K.T.G. and M.J.D.

\section{Author contributions}

E.A.A. designed and performed biochemical and cell-based experiments, analyzed data, assisted in figure preparation, and helped write the paper. R.C. designed and performed biochemical and cell-based experiments, analyzed data, and assisted in figure preparation. S.J., L.A., and D.O. designed, synthesized, and purified custom oligonucleotides and helped prepare figures and edit the manuscript. P.J.J. created and validated HEK + EGFP cells constitutively expressing AsCas12a. M.J.D. and K.T.G. conceived of the project, designed experiments, analyzed data, prepared figures, and wrote the paper.

\section{Competing interests}

The authors declare no competing interests.

\section{Additional information}

Supplementary information The online version contains supplementary material available at https://doi.org/10.1038/s41467-021-26989-z.

Correspondence and requests for materials should be addressed to Masad J. Damha or Keith T. Gagnon.

Peer review information Nature Communications thanks Jean-Paul Desaulniers and the other, anonymous, reviewer(s) for their contribution to the peer review of this work.

Reprints and permission information is available at http://www.nature.com/reprints

Publisher's note Springer Nature remains neutral with regard to jurisdictional claims in published maps and institutional affiliations.

cc (i) Open Access This article is licensed under a Creative Commons Attribution 4.0 International License, which permits use, sharing, adaptation, distribution and reproduction in any medium or format, as long as you give appropriate credit to the original author(s) and the source, provide a link to the Creative Commons license, and indicate if changes were made. The images or other third party material in this article are included in the article's Creative Commons license, unless indicated otherwise in a credit line to the material. If material is not included in the article's Creative Commons license and your intended use is not permitted by statutory regulation or exceeds the permitted use, you will need to obtain permission directly from the copyright holder. To view a copy of this license, visit http://creativecommons.org/ licenses/by/4.0/.

(C) The Author(s) 2021 